\title{
Effect of perfluorosulfonic acid side chains on oxygen permeation in hydrated ionomers of PEMFCs: Molecular dynamics simulation approach
}

\section{Sung Hyun Kwon}

Pusan National University

\section{Haisu Kang}

Pusan National University

\section{Young-Jun Sohn}

Korea Institute of Energy Research

Jinhee Lee

Hyundai Motor (South Korea)

\section{Sunbo Shim}

Hyundai Motor (South Korea)

\section{Seung Geol Lee ( $\nabla$ seunggeol.lee@pusan.ac.kr)}

Pusan National University

\section{Research Article}

Keywords: perfluorosulfonic acid (PFSA) ionomers, polymer electrolyte membrane fuel cells (PEMFCs), molecular dynamics (MD), Nafion, Aquivion, 02 permeability

Posted Date: December 16th, 2020

DOl: https://doi.org/10.21203/rs.3.rs-127966/v1

License: (9) This work is licensed under a Creative Commons Attribution 4.0 International License. Read Full License

Version of Record: A version of this preprint was published at Scientific Reports on April 22nd, 2021. See the published version at https://doi.org/10.1038/s41598-021-87570-8. 


\title{
Effect of perfluorosulfonic acid side chains on oxygen permeation in hydrated ionomers of PEMFCs: Molecular dynamics simulation approach
}

\author{
Sung Hyun Kwon,,$^{1,+}$ Haisu Kang, ${ }^{1,+}$ Young-Jun Sohn, ${ }^{2}$ Jinhee Lee, ${ }^{3}$ \\ Sunbo Shim ${ }^{3, *}$ and Seung Geol Lee ${ }^{1,4, *}$ \\ ${ }^{1}$ School of Chemical Engineering, Pusan National University, 2, Busandaehak-ro \\ 63beon-gil, Geumjeong-gu, Busan 46241, Republic of Korea
}

${ }^{2}$ Fuel Cell Laboratory, Korea Institute of Energy Research (KIER), Yuseong-gu, Daejeon 34129, Republic of Korea

${ }^{3}$ Hyundai Motor Company, 17-5, Mabuk-ro 240, Giheung-gu, Yongin-si, Gyeonggi-do, 16891, Republic of Korea

${ }^{4}$ Department of Organic Material Science and Engineering, Pusan National University, 2, Busandaehak-ro 63beon-gil, Geumjeong-gu, Busan 46241, Republic of Korea

\footnotetext{
${ }^{\dagger}$ Sung Hyun Kwon and Haisu Kang contributed equally to this work.

*Corresponding authors:

sunbo.shim@hyundai.com (S. Shim)

seunggeol.lee@pusan.ac.kr (S.G. Lee)
} 


\begin{abstract}
We prepared two types of perfluorosulfonic acid (PFSA) ionomers with Aquivion (short side chain) and Nafion (long side chain) on a Pt surface and varied their water contents $(2.92 \leq \lambda \leq$ 13.83) to calculate the solubility and permeability of $\mathrm{O}_{2}$ in hydrated PFSA ionomers on a $\mathrm{Pt}$ surface using full atomistic molecular dynamics (MD) simulations. The solubility and permeability of $\mathrm{O}_{2}$ molecules in hydrated Nafion ionomers were greater than those of $\mathrm{O}_{2}$ molecules in hydrated Aquivion ionomers at the same water content, indicating that the permeation of $\mathrm{O}_{2}$ molecules in the ionomers is affected not only by the diffusion coefficient of $\mathrm{O}_{2}$ but also by the solubility of $\mathrm{O}_{2}$. Notably, $\mathrm{O}_{2}$ molecules are more densely distributed in regions where water and hydronium ions have a lower density in hydrated Pt/PFSA ionomers. Radial distribution function (RDF) analysis was performed to investigate where $\mathrm{O}_{2}$ molecules preferentially dissolve in PFSA ionomers on a $\mathrm{Pt}$ surface. The results showed that $\mathrm{O}_{2}$ molecules preferentially dissolved between hydrophilic and hydrophobic regions in a hydrated ionomer. The RDF analysis was performed to provide details of the $\mathrm{O}_{2}$ location in hydrated PFSA ionomers on a $\mathrm{Pt}$ surface to evaluate the influence of $\mathrm{O}_{2}$ solubility in ionomers with side chains of different lengths. The coordination number of $\mathrm{C}($ center $)-\mathrm{O}_{2}\left(\mathrm{O}_{2}\right)$ and $\mathrm{O}($ side chain $)-\mathrm{O}\left(\mathrm{O}_{2}\right)$ pairs in hydrated Nafion ionomers was higher than that of the same pairs in hydrated Aquivion ionomers with the same water content. Our investigation provides detailed information about the properties of $\mathrm{O}_{2}$ molecules in different PFSA ionomers on a $\mathrm{Pt}$ surface and with various water contents, potentially enabling the design of better-performing PFSA ionomers for use in polymer electrolyte membrane fuel cells.
\end{abstract}

Keywords: perfluorosulfonic acid (PFSA) ionomers, polymer electrolyte membrane fuel cells (PEMFCs), molecular dynamics (MD), Nafion, Aquivion, $\mathrm{O}_{2}$ permeability 


\section{Introduction}

Polymer electrolyte membrane fuel cells (PEMFCs) are environmentally friendly energy sources that can alleviate environmental problems because of their low emissions of environmental pollutant gases such as $\mathrm{SO}_{x}, \mathrm{NO}_{x}, \mathrm{CO}_{2}$, and CO. ${ }^{1,2}$ PEMFCs have been used in various applications such as fuel-cell vehicles and power supplies (including portable power supplies) because, in addition to their ecofriendly benefits, PEMFCs can also generate high power densities and operate with short start-up times because of their low operating temperature. ${ }^{1,3-5}$ In general, PEMFCs consist of membrane electrode assembly (MEA) layers, gas-diffusion layers, microporous layers, gas flow channels, and bipolar plates. ${ }^{3}$ The MEA layers are especially important because the cell performance and durability of PEMFCs are strongly affected by the design and composition of their MEA layers. ${ }^{4}$ MEA layers in a PEMFC system comprise catalyst layers (CLs) and a polymer membrane; the polymer membrane plays a critical role in transferring protons from the anode to the cathode in the process of generating electricity. ${ }^{5}$ CLs also play a critical role because the electrochemical reactions related to energy conversion in a PEMFC, such as the hydrogen oxidation reaction or oxygen reduction reaction (ORR), occur in CLs. The structure of CLs includes a carbon matrix with a Pt catalyst $(\mathrm{Pt} / \mathrm{C})$ and proton-conducting ionomers. Importantly, the proton transfer performance of a $\mathrm{CL}$ is affected by its ionomer thin film on $\mathrm{Pt} / \mathrm{C}$ because protons can directly reach the Pt surface through the hydrated ionomer thin film. Therefore, the composition and morphology of the ionomers strongly influence the performance of a PEMFC.

The proton-conducting ionomers in PEMFCs are categorized as a perfluorinated acid (PFSA), nonfluorinated hydrocarbon, or an acid-base complex. ${ }^{1}$ Among these various polymer ionomers, PFSA ionomers such as Nafion (DuPont), ${ }^{6,7}$ Aquivion (Dow Chemical), ${ }^{8,9}$ AciplexS (Asahi Glass), [10] and Flemion (Asahi Glass) ${ }^{10,11}$ have been extensively used in PEMFCs because of their excellent proton conductivity and good mechanical, chemical, and thermal stabilities. ${ }^{12}$ PFSA-based ionomers are composed of main chains of polytetrafluoroethylene (PTFE) and side chains terminated by sulfonic acid groups. Both experimental ${ }^{13-15}$ and theoretical ${ }^{16-24}$ investigations have been performed to elucidate the proton transfer and $\mathrm{O}_{2}$ permeation mechanisms in PEMFC systems because the length of the side chains in PFSA ionomers affects PEMFC performance. 
Garsany et al. ${ }^{13}$ and Siracusano et al. ${ }^{14}$ experimentally investigated PFSA ionomersspecifically, Aquivion, which has a short side chain, and Nafion, which has a long side chain - to improve PEMFC performance. They concluded that Aquivion exhibits better cell performance than Nafion in PEMFCs because the Aquivion ionomer in the cathode CLs has a lower proton transport resistance, lower charge transfer resistance for the ORR, and lower mass transport resistance than the Nafion ionomer. Baschetti et al. ${ }^{15}$ investigated gas permeation in Nafion and Aquivion ionomers at various temperatures and relative humidities. Humidity and temperature have especially strong effects on gas permeability, and Nafion 117 ionomer was found to exhibit greater $\mathrm{O}_{2}$ gas permeability than Aquivion at $50 \square$.

Several groups have investigated the relationship between the diffusion coefficients of water and hydronium ions and PFSA morphologies in systems with various water contents and at different temperatures using molecular dynamics (MD) simulations. ${ }^{16-21}$ The diffusion coefficients of water and hydronium ions were found to increase with increasing PFSA water content and increasing temperature. In addition, the sulfur-sulfur interatomic distance in PFSA increased with increasing PFSA water content. MD simulations ${ }^{22-24}$ have also been performed to investigate $\mathrm{O}_{2}$ permeation in Nafion ionomers in CLs. Kurihara et al. ${ }^{22,23}$ investigated the permeation of $\mathrm{O}_{2}$ gas into a Nafion ionomer on a Pt surface using MD simulations because such simulations are useful for understanding the nanoscale structures in the CLs of PEMFCs. They concluded that the diffusion coefficient of $\mathrm{O}_{2}$ molecules increased and the solubility of $\mathrm{O}_{2}$ molecules decreased with increasing water content in the Nafion ionomer. Jinnouchi et al. ${ }^{24}$ also used MD simulations to investigate $\mathrm{O}_{2}$ permeation through a Nafion thin film on a Pt surface, where the water content of the Nafion film was varied. Their results indicated that $\mathrm{O}_{2}$ permeation in Nafion increased with increasing water content and that understanding the behavior of $\mathrm{O}_{2}$ in PFSA ionomers on a $\mathrm{Pt}$ surface is critical to understanding its permeation properties. Therefore, the aforementioned experimental results indicate that the length of the side chain in PFSA ionomers can affect both the performance of PEMFCs and the $\mathrm{O}_{2}$ permeation behavior. Therefore, studies comparing of the $\mathrm{O}_{2}$ permeation properties of Nafion and Aquivion are needed to elucidate the effect of side-chain length in PFSA ionomers in PEMFCs.

In the present study, computational simulations using the full atomistic MD simulation technique are carried out to obtain detailed molecular information for calculating the 
transport properties of hydrated PFSA ionomers with various water contents on a Pt surface. In addition, the $\mathrm{O}_{2}$ permeation properties of hydrated PFSA with different side-chain lengths were measured at the interfacial region on the Pt surface. Therefore, two types of PFSA ionomers-Nafion (longer side chain) and Aquivion (shorter side chain) - were prepared for measurement of the $\mathrm{O}_{2}$ permeability at various water contents, enabling the relationship between the hydrated PFSA structure and the $\mathrm{O}_{2}$ permeation properties to be elucidated. In addition, the distribution of $\mathrm{O}_{2}$ and water in PFSA ionomers on a Pt surface were also analyzed using density profiles and radial distribution functions (RDFs) with various water contents at the operating temperature of a PEMFC $(353.15 \mathrm{~K})$. We expect that the results of this study will provide detailed information about $\mathrm{O}_{2}$ permeability of water-containing PFSA ionomers on a Pt surface and can provide guidance for the design of PFSA ionomers for use in PEMFCs.

\section{Computational Details}

\subsection{Model preparation}

Figure 1 shows the chemical structures of the Nafion and Aquivion ionomers. Each ionomer was composed such that each polymer had 10 repeat units with 10 sulfonic acid groups per polymer chain. The molecular weight of the Nafion and Aquivion polymers was 9969.83 $\mathrm{g} / \mathrm{mol}$ and $8309.63 \mathrm{~g} / \mathrm{mol}$ per polymer chain; equivalent weights (EWs) of $\sim 1000 \mathrm{~g} / \mathrm{mol}$ and $\sim 830 \mathrm{~g} / \mathrm{mol}$ were applied, respectively. Water, $\mathrm{O}_{2}$ molecules, and hydronium ions were prepared for constructing hydrated PFSA ionomers. The components of each PEMFC system are summarized in Table 1.

\subsection{Force-field and MD parameters}

To describe inter- and intramolecular interactions in the Nafion and Aquivion in PEMFC systems, we applied a modified DREIDING force field ${ }^{25}$ in our simulations. The DREIDING force field has been widely used to describe PEMFCs systems. ${ }^{26-29}$ The force fields of water molecules and Pt atoms were applied using F3C force field ${ }^{30}$ and the embedded-atom method (EAM) force field, respectively. ${ }^{31}$ The total potential energy $E_{\text {total }}$ in PEMFC systems can be calculated using to $\mathrm{Eq}(1)$ : 


$$
E_{\text {total }}=E_{\mathrm{vdW}}+E_{\mathrm{Q}}+E_{\text {bond }}+E_{\text {angle }}+E_{\text {torsion }}+E_{\text {inversion }}+E_{\mathrm{EAM}}
$$

where $E_{\mathrm{vdW}}, E_{\mathrm{Q}}, E_{\mathrm{bond}}, E_{\text {angle }}, E_{\text {torsion }}, E_{\text {inversion, }}$ and $E_{\mathrm{EAM}}$ are the van der Waals, electrostatic, bond-stretching, angle-bending, torsion, inversion, and the EAM energies, respectively. For calculating entire MD simulations for PEMFC systems, the large-scale atomic/molecular massively parallel simulator (LAMMPS) $\operatorname{code}^{32,33}$ from Plimpton at Sandia was used. All MD simulations were carried out using the velocity Verlet algorithm ${ }^{34}$ to integrate equations of atomic motion, with a time steps of $1 \mathrm{fs}$. The electrostatic interactions in our systems were calculated using the particle-particle particle-mesh method. ${ }^{35}$ The charges of particles in Nafion and Aquivion were calculated via density functional theory (DFT) calculations using the Mulliken charge analysis method ${ }^{36}$ in the Materials Studio software. ${ }^{37}$ All DFT calculations for charge analyses were carried out using the double numerical basis set with polarization (DNP) function and the generalized gradient approximation with the Perdew-Burke-Ernzerhof functional. ${ }^{38}$

\subsection{Force-field parameters between PEMFC components and the Pt surface}

We used the nonbonded interaction energies reported by Brunello et al. ${ }^{39}$ to describe the interactions of Pt atoms with Nafion, Aquivion, water, and hydronium ions. In addition, for the interaction energies between $\mathrm{O}_{2}$ and a Pt slab, we calculated van der Waals parameters via DFT calculations to describe detailed intermolecular interactions using a Pt (111) slab with three atomic layers with periodic boundary conditions (PBCs) of $8.324 \times 8.324 \times 25.000 \AA^{3}$, as shown in Figure 2. The DFT calculation details were the same as those used in the charge analysis (section 2.2.1), and a semi-empirical dispersion correction (DFT-D) with the Tkatchenko-Scheffler scheme was additionally applied. ${ }^{40}$ Band-structure calculations with $k$ points were performed with a $4 \times 4 \times 1$ Monkhorst-Pack $k$-point mesh. ${ }^{41}$

\subsection{Model construction}

To generate the Pt (111) surface and hydrated PFSA ionomers, we constructed a Pt (111) slab with five atomic layers with PBCs of $38.446 \times 38.845 \times 300.000 \AA^{3}$. A $z$-direction length of $300 \AA$ was used to prevent interaction beyond the PBCs. To construct hydrated PFSA ionomers on a Pt (111) surface, the $\lambda$ (water molecules per sulfonic acid group) values were 2.92, 6.15, 9.77, and 13.83 for hydrated Nafion and Aquivion ionomers. The PFSA ionomers were composed of six polymer chains with 60 hydronium ions to maintain electrical 
neutrality. The Monte Carlo simulation in the Amorphous Cell module of the Materials Studio software ${ }^{37}$ was used to construct the initial configuration of hydrated PFSA ionomers on a Pt (111) surface.

\subsection{MD simulations}

After the initial hydrated PFSA with Pt (111) surface models were constructed, the temperature was gradually increased to the operating temperature $(353.15 \mathrm{~K})$ of PEMFCs with canonical ensemble (NVT) for 1 ns. Consequently, 15 ns MD simulations were performed by NVT simulation to obtain the equilibrated structures. To simulate the permeation of $\mathrm{O}_{2}$ into PFSA ionomers, $735 \mathrm{O}_{2}$ molecules with a thickness of $100 \AA$ were placed on the equilibrated PFSA ionomers. After the $\mathrm{O}_{2}$ molecules were added, a total of 150 ns of NVT simulations at $353.15 \mathrm{~K}$ was performed. The last $10 \mathrm{~ns}$ of NVT simulation was used for data collection of the $\mathrm{O}_{2}$ permeation properties.

\section{Results and Discussion}

\section{1 van der Waals parameters for $\mathrm{O}_{2}$ and $\mathrm{Pt}$}

For a better description of the $\mathrm{O}_{2}$ permeation process inside a hydrated Nafion ionomer thin film on a Pt (111) surface, the atomic interaction curve between $\mathrm{O}_{2}$ and the Pt surface was reproduced by DFT under the framework of the DREIDING force field. Figure 2(a) shows $\mathrm{O}_{2}$ adsorbed onto the Pt surface, which was built for calculation of the adsorption energy as a function of the $z$-distance. The calculated adsorption energy as a function of distance was fitted to the Lennard-Jones potential in Figure 2(b), which well reproduced the results of DFT calculations. The Lennard-Jones potential function is shown in Eq (2):

$$
E=\varepsilon\left[\left(\frac{r_{\mathrm{m}}}{r}\right)^{12}-2\left(\frac{r_{\mathrm{m}}}{r}\right)^{6}\right]
$$

where $E$ indicates the potential energy with changing distance $r$ and $\varepsilon$ is the depth of the potential well at distance $r_{\mathrm{m}}$. The values of $\varepsilon$ and $r_{\mathrm{m}}$ for the oxygen atoms in $\mathrm{O}_{2}$ molecules on a Pt surface are $4.070 \mathrm{kcal} / \mathrm{mol}$ and $2.338 \AA$, respectively. The fitted interaction well describes detailed interactions that the DREIDING force field cannot describe. Thus, the 
fitted interaction between $\mathrm{O}_{2}$ and $\mathrm{Pt}$ surfaces was used in the MD simulation to analyze the process of $\mathrm{O}_{2}$ permeation into a Nafion ionomer coated onto a Pt surface.

\subsection{Equilibrated structure}

Equilibrated structures in Figure 3(a)-(h) were obtained from the data corresponding to the final $5 \mathrm{~ns}$ of the MD trajectories. Figure 3(a)-(d) show a hydrated Nafion thin film on a Pt surface, and Figure 3(e)-(h) show a hydrated Aquivion thin film on a Pt surface, both under different hydration levels, $\lambda$. As $\lambda$ increases, the Nafion and Aquivion films become gradually segregated into hydrophilic water clusters and hydrophobic regions with PTFE backbones of Nafion and Aquivion ionomer, respectively. Water molecules are predominantly adsorbed as a thin layer onto the Pt surface because they strongly interact with this surface. ${ }^{29}$

To quantitatively analyze the permeation of $\mathrm{O}_{2}$ molecules, we investigated the density profile of hydrated Nafion, water molecules with hydronium ions, and $\mathrm{O}_{2}$ molecules, as shown in Figure 4(a)-(d). Dotted lines represent the average density of a bulk Nafion membrane, as reported in our previous study, ${ }^{20}$ which indicates that the density of hydrated Nafion on a Pt surface is not substantially different from that of a bulk membrane except for the density at the Nafion-Pt interface. As shown in the equilibrated structures in Figure 3(a)-(h), hydrated Nafion ionomers and water molecules with hydronium ions exhibit the highest density at the hydrated Nafion-Pt interface because of their strong attractive interaction. The purple line indicates the point of $\mathrm{O}_{2}$ solvation from the hydrated Nafion-gas interface, which is determined by the distance corresponding to the average number of total solvated $\mathrm{O}_{2}$ molecules. At this point, the density of $\mathrm{O}_{2}$ molecules abruptly decreases from its maximum value. These results are in good agreement with those of Jinnouchi et al. ${ }^{24}$, who reported that an energy barrier at the Nafion-gas interface dominates the solubility of $\mathrm{O}_{2}$ in hydrated Nafion. Inside hydrated Nafion, dissolved $\mathrm{O}_{2}$ molecules exhibit the highest density and water molecules with hydronium ions exhibit the lowest density. This trend becomes more discernible as the hydration level increases. These results suggest that $\mathrm{O}_{2}$ molecules are not preferentially positioned inside hydrophilic domains but rather at the interfacial regions between hydrophobic and hydrophilic regions.

We also investigated the density profile of water molecules, hydronium ions, and $\mathrm{O}_{2}$ molecules in hydrated Aquivion; the results are shown in Figure 5(a)-(d). Like Nafion, 
hydrated Aquivion and water molecules with hydronium ions show the highest density at the Aquivion-Pt interface because of their strong attractive interaction. The hydrated Aquivion is thinner than the hydrated Nafion because of Aquivion's shorter side chains and lower EW. The density of $\mathrm{O}_{2}$ molecules also abruptly decreases the maximum value at the distance indicated by the purple line, which represents the distance at which $\mathrm{O}_{2}$ solvation begins. Inside hydrated Aquivion, the dissolved $\mathrm{O}_{2}$ molecules exhibit the highest density and water molecules with hydronium ions exhibit the lowest density.

\section{$3.3 \mathrm{O}_{2}$ solubility and permeation}

To quantify the solvation of $\mathrm{O}_{2}$ molecules by hydration level, we calculated the solubility on the basis of the average number of dissolved $\mathrm{O}_{2}$ molecules at the distance indicated by the purple vertical line in Figures 4 and 5. The solubility of Nafion and Aquivion ionomers as a function of the hydration level is presented in Figure 6(a). The solubility of $\mathrm{O}_{2}$ decreases with increasing hydration level in both the Nafion and Aquivion ionomers. Because the interfacial region between hydrophilic and hydrophobic domains becomes relatively limited when the net ionomer volume increases under hydration, the number of sites available for dissolved $\mathrm{O}_{2}$ molecules also becomes limited. Therefore, the ionomers exhibit low $\mathrm{O}_{2}$ solubility at high hydration levels $(\lambda)$. At the same $\lambda$, the $\mathrm{O}_{2}$ solubility is higher in the Nafion ionomer than in the Aquivion ionomer, which means that the solvation of $\mathrm{O}_{2}$ molecules in the Aquivion ionomer is restricted compared with that in the Nafion ionomer.

Using the solubility values, we derived the permeability coefficient of $\mathrm{O}_{2}$ molecules. Gases permeate through ionomers via a solution-diffusion mechanism, where the dissolved gas molecules diffuse into the ionomers. ${ }^{42}$ Thus, the permeability coefficient $P$ is described by the equation

$$
P=D \times S
$$

where $D$ is the diffusion coefficient and $S$ is solubility. The diffusion coefficient in our system was obtained from the self-diffusion coefficient of $\mathrm{O}_{2}$ molecules in the bulk structures of Nafion and Aquivion. The calculated permeability coefficients for $\mathrm{O}_{2}$ molecules in Nafion and Aquivion ionomer are shown in Figure 6(b); these values are consistent with those reported by Baschetti et al. ${ }^{15}$. With increasing hydration level $\lambda$, the permeability of $\mathrm{O}_{2}$ in both Nafion and Aquivion ionomers increases until $\lambda=9.77$. At $\lambda>9.77$, the permeability 
increases slightly because, despite the diminished solubility, the self-diffusion coefficient of $\mathrm{O}_{2}$ in a bulk ionomer membrane increases with increasing hydration level; thus, permeability increases with increasing hydration level. In our previous work, ${ }^{20}$ we reported that $\mathrm{O}_{2}$ molecules exhibit greater diffusion in Aquivion ionomers than in Nafion ionomers because of the Aquivion ionomers' better-developed water channels. However, at the same hydration level $\lambda$, both the permeability and solubility of $\mathrm{O}_{2}$ are higher in Nafion ionomer than in Aquivion ionomer. This result means that solubility is more critical to the permeability, which is dominated by the availability of solvation sites for dissolved $\mathrm{O}_{2}$ molecules in the ionomer.

\subsection{RDF analysis}

The interface region between hydrophilic and hydrophobic domains in a hydrated PFSA ionomer, where $\mathrm{O}_{2}$ molecules preferentially dissolve, is most likely to be the side-chain part above the sulfonic acid groups. That is, $\mathrm{O}_{2}$ solvation mainly occurs at the side chains of the ionomers. Thus, the difference in solubility between Nafion and Aquivion is reasonably deduced to arise from their different side-chain structures. In this regard, we analyzed the structure between $\mathrm{O}_{2}$ and the main component of the Nafion and Aquivion side-chain structures to understand the difference in $\mathrm{O}_{2}$ solubility between them. As shown in Figure 7(a), we analyzed correlations between Carbon(center)-Oxygen $\left(\mathrm{O}_{2}\right)$ and Oxygen(side chain)-Oxygen $\left(\mathrm{O}_{2}\right)$ using RDFs. The RDF of each pair is described by the following equation:

$$
g_{A-B}(r)=\left(\frac{n_{B}}{4 \pi r^{2} d r}\right) /\left(\frac{N_{B}}{V}\right)
$$

where $n_{B}$ is the number of $B$ particles located at a distance $r$ in a shell of thickness $d r$ from particle $A, N_{B}$ is the number of $B$ particles in the system, and $V$ is the total volume of the system; $N_{B} / V$ can be represented by the number density, $\rho$. The $\rho g(r)$ of each pair of Nafion and Aquivion ionomers is presented in Figure 7(b) and (c), respectively. The $\rho g(r)$ of both $\mathrm{C}($ center $)-\mathrm{O}\left(\mathrm{O}_{2}\right)$ and $\mathrm{O}($ side chain $)-\mathrm{O}\left(\mathrm{O}_{2}\right)$ shows the first peak at $7.87 \AA$ and $8.51 \AA$, respectively. These values decrease with increasing hydration level. At the same hydration level, the $\rho g(r)$ of both $\mathrm{C}($ center $)-\mathrm{O}\left(\mathrm{O}_{2}\right)$ and $\mathrm{O}\left(\right.$ side chain) $-\mathrm{O}\left(\mathrm{O}_{2}\right)$ is greater in the Nafion ionomer than in the Aquivion ionomer. This result means that $\mathrm{O}_{2}$ has a more favorable correlation with $\mathrm{C}$ (center) and $\mathrm{O}$ (side chain) in the Nafion ionomer than with those in the 
Aquivion ionomer. The first coordination number $(\mathrm{CN})$ at the distance of the first peak was calculated (Table 2). As shown in Figure 7(d) and (e), the $\mathrm{CN}$ of $\mathrm{C}($ center $)-\mathrm{O}\left(\mathrm{O}_{2}\right)$ and $\mathrm{O}($ side chain) $-\mathrm{O}\left(\mathrm{O}_{2}\right)$ pairs also decreases as the hydration level increases with increasing $\rho g(r)$. At the same hydration level, the Nafion ionomer shows a higher $\mathrm{CN}$ of each pair than the Aquivion ionomer. This result suggests that more $\mathrm{O}_{2}$ molecules are coordinated to the side chain of the Nafion ionomer than to that of the Aquivion ionomer, especially to the side chains' oxygen atoms, which results in greater solubility of $\mathrm{O}_{2}$ in the Nafion ionomer. In addition, we note that that Aquivion shows a similar $\mathrm{CN}$ as Nafion at $\lambda=2.92$. As described in the previous section, we also observed that the difference in solubility and permeability is more discernible at a hydration level greater than $\lambda=2.92$. At a low hydration level, the site for $\mathrm{O}_{2}$ solvation is uncertain because of a lack of phase segregation. However, as the hydration level increases, the solvation sites of $\mathrm{O}_{2}$ molecules become limited to the side-chain region of the ionomer. Consequently, Nafion and Aquivion exhibit an observable difference in solubility at higher hydration levels.

\section{Conclusion}

We prepared two types of hydrated PFSA ionomers on a Pt (111) surface and varied their water contents $(2.92 \leq \lambda \leq 13.83)$ to analyze the distribution morphologies and permeability properties of $\mathrm{O}_{2}$ molecules in detail using full atomistic MD simulations at the operating temperature of PEMFCs $(353.15 \mathrm{~K})$. The hydrated PFSA ionomer structures were prepared using Nafion (long side chain) and Aquivion (short side chain). In the MD simulations, $\mathrm{O}_{2}$ molecules were gradually permeated into hydrated PFSA ionomers on the Pt surface. Density profile analysis for hydrated PFSA ionomers was performed to calculate the $\mathrm{O}_{2}$ solubility in hydrated PFSA ionomers with various $\lambda$. The $\mathrm{O}_{2}$ solubility in hydrated Nafion ionomers on the Pt surface was higher than that in hydrated Aquivion ionomers on the Pt surface at the same $\lambda$ values. The RDF analysis showed that the first $\mathrm{CN}$ of $\mathrm{C}($ center $)-\mathrm{O}\left(\mathrm{O}_{2}\right)$ and $\mathrm{O}($ side chain)-O $\left(\mathrm{O}_{2}\right)$ pairs of hydrated Nafion ionomers was greater than that of hydrated Aquivion ionomers at the same water content. These results suggest that more $\mathrm{O}_{2}$ molecules are coordinated to the side chains of the Nafion ionomer than to those of the Aquivion ionomer, especially oxygen atoms, which results in greater $\mathrm{O}_{2}$ solubility in the Nafion ionomer. 


\section{Acknowledgements}

This work was supported by the R\&D Collaboration Programs of Hyundai Motor Company. This research was supported by the National Research Foundation of Korea (NRF) funded by the Ministry of Science, ICT \& Future Planning (No. 2020M1A2A2080807). This research was supported by Global Frontier Program through the Global Frontier Hybrid Interface Materials (GFHIM) of the National Research Foundation of Korea (NRF) funded by the Ministry of Science, ICT and Future Planning (No. 2013M3A6B1078882). 


\section{References}

1 Peighambardoust, S. J., Rowshanzamir, S. \& Amjadi, M. Review of the proton exchange membranes for fuel cell applications. Int J Hydrogen Energ 35, 9349-9384, (2010).

2 Stambouli, A. B. Fuel cells: The expectations for an environmental-friendly and sustainable source of energy. Renewable and Sustainable Energy Reviews 15, 4507-4520, (2011). Debe, M. K. Electrocatalyst approaches and challenges for automotive fuel cells. Nature 486, 43-51, (2012).

4 Kraytsberg, A. \& Ein-Eli, Y. Review of Advanced Materials for Proton Exchange Membrane Fuel Cells. Energ Fue/ 28, 7303-7330, (2014).

5 Wang, Y., Chen, K. S., Mishler, J., Cho, S. C. \& Adroher, X. C. A review of polymer electrolyte membrane fuel cells: Technology, applications, and needs on fundamental research. App/ Energ 88, 981-1007, (2011).

6 Gierke, T. D., Munn, G. E. \& Wilson, F. C. The Morphology in Nafion Perfluorinated Membrane Products, as Determined by Wide-Angle and Small-Angle X-Ray Studies. J Polym Sci Pol Phys 19, 1687-1704, (1981).

7 Kariduraganavar, M. Y., Nagarale, R. K., Kittur, A. A. \& Kulkarni, S. S. Ion-exchange membranes: preparative methods for electrodialysis and fuel cell applications. Desalination 197, 225-246, (2006).

8 Moore, R. B. \& Martin, C. R. Morphology and Chemical-Properties of the Dow Perfluorosulfonate Ionomers. Macromolecules 22, 3594-3599, (1989).

9 Gebel, G. \& Moore, R. B. Small-angle scattering study of short pendant chain perfuorosulfonated ionomer membranes. Macromolecules 33, 4850-4855, (2000).

10 Yoshida, N., Ishisaki, T., Watakabe, A. \& Yoshitake, M. Characterization of Flemion (R) membranes for PEFC. Electrochim Acta 43, 3749-3754, (1998).

11 Saito, M., Arimura, N., Hayamizu, K. \& Okada, T. Mechanisms of ion and water transport in perfluorosulfonated ionomer membranes for fuel cells. J Phys Chem B 108, 16064-16070, (2004).

12 Smitha, B., Sridhar, S. \& Khan, A. A. Solid polymer electrolyte membranes for fuel cell applications - a review. J Membrane Sci 259, 10-26, (2005).

13 Garsany, Y. et al. Improving PEMFC Performance Using Short-Side-Chain Low-EquivalentWeight PFSA Ionomer in the Cathode Catalyst Layer. J Electrochem Soc 165, F381-F391, (2018).

14 Siracusano, S. et al. Performance analysis of short-side-chain Aquivion (R) perfluorosulfonic acid polymer for proton exchange membrane water electrolysis. J Membrane Sci 466, 1-7, (2014).

15 Baschetti, M. G., Minelli, M., Catalano, J. \& Sarti, G. C. Gas permeation in perflurosulfonated membranes: Influence of temperature and relative humidity. Int $J$ Hydrogen Energ 38, 11973-11982, (2013). 
Jang, S. S., Molinero, V., Çağın, T. \& Goddard, W. A. Nanophase-Segregation and Transport in Nafion 117 from Molecular Dynamics Simulations: Effect of Monomeric Sequence. $J$ Phys Chem B 108, 3149-3157, (2004).

17 Cui, S. T. et al. A molecular dynamics study of a nafion polyelectrolyte membrane and the aqueous phase structure for proton transport. J Phys Chem B 111, 2208-2218, (2007).

18 Venkatnathan, A., Devanathan, R. \& Dupuis, M. Atomistic simulations of hydrated Nafion and temperature effects on hydronium ion mobility. J Phys Chem B 111, 7234-7244, (2007).

19 Sunda, A. P. \& Venkatnathan, A. Molecular dynamics simulations of side chain pendants of perfluorosulfonic acid polymer electrolyte membranes. J Mater Chem A 1, 557-569, (2013).

20 Kwon, S. $\mathrm{H}$. et al. Investigating the influence of the side-chain pendants of perfluorosulfonic acid membranes in a PEMFC by molecular dynamics simulations. Materials Today Communications 21, 100625, (2019).

21 Paddison, S. J. \& Elliott, J. A. Molecular Modeling of the short-side-chain perfluorosulfonic acid membrane. J Phys Chem A 109, 7583-7593, (2005).

22 Kurihara, Y., Mabuchi, T. \& Tokumasu, T. Molecular Analysis of Structural Effect of Ionomer on Oxygen Permeation Properties in PEFC. J Electrochem Soc 164, F628-F637, (2017).

23 Kurihara, Y., Mabuchi, T. \& Tokumasu, T. Molecular dynamics study of oxygen transport resistance through ionomer thin film on Pt surface. J Power Sources 414, 263-271, (2019).

24 Jinnouchi, R., Kudo, K., Kitano, N. \& Morimoto, Y. Molecular Dynamics Simulations on O-2 Permeation through Nafion lonomer on Platinum Surface. Electrochim Acta 188, 767-776, (2016).

25 Mayo, S. L., Olafson, B. D. \& Goddard, W. A. Dreiding - a Generic Force-Field for Molecular Simulations. J Phys Chem-Us 94, 8897-8909, (1990).

26 Devanathan, R., Venkatnathan, A. \& Dupuis, M. Atomistic simulation of nafion membrane. 2. Dynamics of water molecules and hydronium ions. J Phys Chem B 111, 13006-13013, (2007).

27 Kwon, S. H., Lee, S. Y., Kim, H.-J., Kim, H.-T. \& Lee, S. G. Molecular Dynamics Simulation to Reveal Effects of Binder Content on Pt/C Catalyst Coverage in a High-Temperature Polymer Electrolyte Membrane Fuel Cell. ACS Applied Nano Materials 1, 3251-3258, (2018).

28 Lee, K. A. et al. Post-assembly modification of polymeric composite membranes using spin drying for fuel cell applications. J Mater Chem A 7, 7380-7388, (2019).

29 Kang, H. et al. Nanostructures of Nafion Film at Platinum/Carbon Surface in Catalyst Layer of PEMFC: Molecular Dynamics Simulation Approach. Journal of Physical Chemistry C 124, 21386-21395, (2020).

30 Levitt, M., Hirshberg, M., Sharon, R., Laidig, K. E. \& Daggett, V. Calibration and testing of a water model for simulation of the molecular dynamics of proteins and nucleic acids in solution. J Phys Chem B 101, 5051-5061, (1997). 
31 Zhou, X. W., Johnson, R. A. \& Wadley, H. N. G. Misfit-energy-increasing dislocations in vapor-deposited CoFe/NiFe multilayers. Phys Rev B 69, (2004).

32 Plimpton, S. Fast Parallel Algorithms for Short-Range Molecular-Dynamics. J Comput Phys 117, 1-19, (1995).

33 Plimpton, S., Pollock, R. \& Stevens, M. in PPSC.

34 Swope, W. C., Andersen, H. C., Berens, P. H. \& Wilson, K. R. A Computer-Simulation Method for the Calculation of Equilibrium-Constants for the Formation of Physical Clusters of Molecules - Application to Small Water Clusters. J Chem Phys 76, 637-649, (1982).

35 Hockney, R. W. \& Eastwood, J. W. Computer simulation using particles. (crc Press, 1988).

36 Mulliken, R. S. Electronic Population Analysis on LCAO-MO Molecular Wave Functions. I. J Chem Phys 23, 1833-1840, (1955).

37 BIOVIA. Materials Studio 2019. San Diego, Dassault Systèmes (2019).

38 Perdew, J. P., Burke, K. \& Ernzerhof, M. Generalized Gradient Approximation Made Simple. Physical Review Letters 77, 3865-3868, (1996).

39 Brunello, G. F. et al. Interactions of Pt nanoparticles with molecular components in polymer electrolyte membrane fuel cells: multi-scale modeling approach. RSC Advances 6, 69670-69676, (2016).

40 Tkatchenko, A. \& Scheffler, M. Accurate Molecular Van Der Waals Interactions from Ground-State Electron Density and Free-Atom Reference Data. Physical Review Letters 102, (2009).

41 Monkhorst, H. J. \& Pack, J. D. Special Points for Brillouin-Zone Integrations. Phys Rev B 13, 5188-5192, (1976).

42 Kusoglu, A. \& Weber, A. Z. New Insights into Perfluorinated Sulfonic-Acid lonomers. Chem Rev 117, 987-1104, (2017). 
Table 1. Details of parameters for the MD simulations.

\begin{tabular}{ccccc}
\hline & $\lambda=\mathbf{2 . 9 2}$ & $\lambda=\mathbf{6 . 1 5}$ & $\lambda=\mathbf{9 . 7 7}$ & $\lambda=\mathbf{1 3 . 8 3}$ \\
\hline No. of water molecules & 115 & 309 & 526 & 770 \\
No. of hydronium ions & 60 & 60 & 60 & 60 \\
$\begin{array}{c}\text { No. of } \mathrm{O}_{2} \text { molecules } \\
\text { No. of PFSA chains (DP 10) } \\
\text { (Nafion or Aquivion) }\end{array}$ & & 735 & & \\
\hline
\end{tabular}


Table 2. The coordination numbers of Nafion and Aquivion on a Pt surface at $353.15 \mathrm{~K}$, where the water contents $(\lambda)$ were varied.

\begin{tabular}{cccccc}
\hline Pairs & Ionomer & $\lambda=\mathbf{2 . 9 2}$ & $\lambda=\mathbf{6 . 1 5}$ & $\lambda=\mathbf{9 . 7 7}$ & $\lambda=\mathbf{1 3 . 8 3}$ \\
\hline $\begin{array}{c}\mathrm{C}(\text { center })-\mathrm{O}\left(\mathrm{O}_{2}\right) \\
(7.87 \AA)\end{array}$ & Nafion & 6.18 & 5.68 & 5.35 & 5.15 \\
\hline $\begin{array}{c}\text { O(side chain) }-\mathrm{O}\left(\mathrm{O}_{2}\right) \\
(8.51 \AA)\end{array}$ & Nafion & 7.56 & 6.60 & 5.94 & 5.64 \\
\hline
\end{tabular}


(a) $\left.f\left(\mathrm{CF}_{2}-\mathrm{CF}_{2}\right)_{5} \mathrm{CF}_{2}-\mathrm{CF}-\mathrm{CF}_{2}\right\}_{\mathrm{n}}$

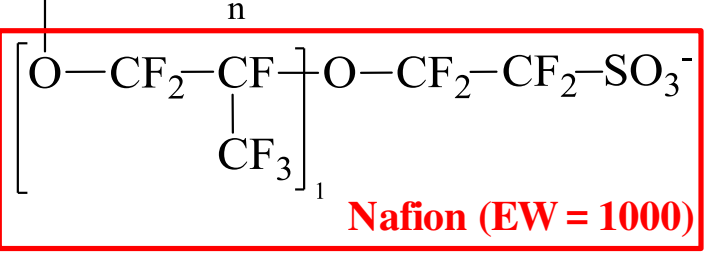

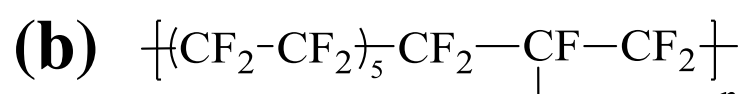

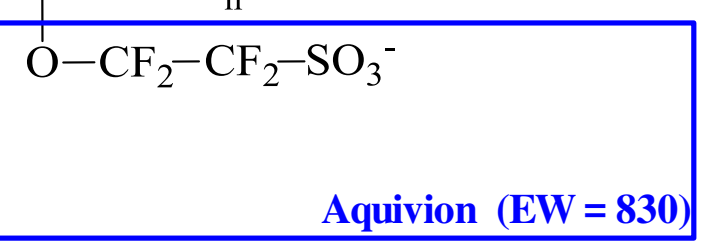

(c)

$$
\mathrm{H}^{-\mathrm{O}}{ }_{\mathrm{H}}
$$

(d)

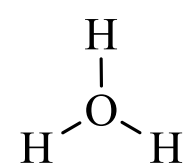

(e)

$$
\mathrm{O}=\mathrm{O}
$$

Figure 1. Chemical structures of PFSA component at (a) ionized Nafion $(E W=1000),(b)$ ionized Aquivion $(E W=830)$, and $(c)$ water, $(d)$ hydronium ion, and (e) $\mathrm{O}_{2}$ molecules. 
(a)

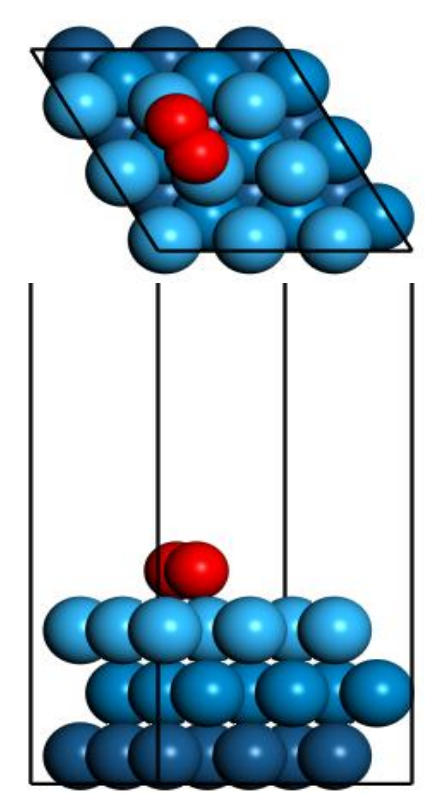

(b)

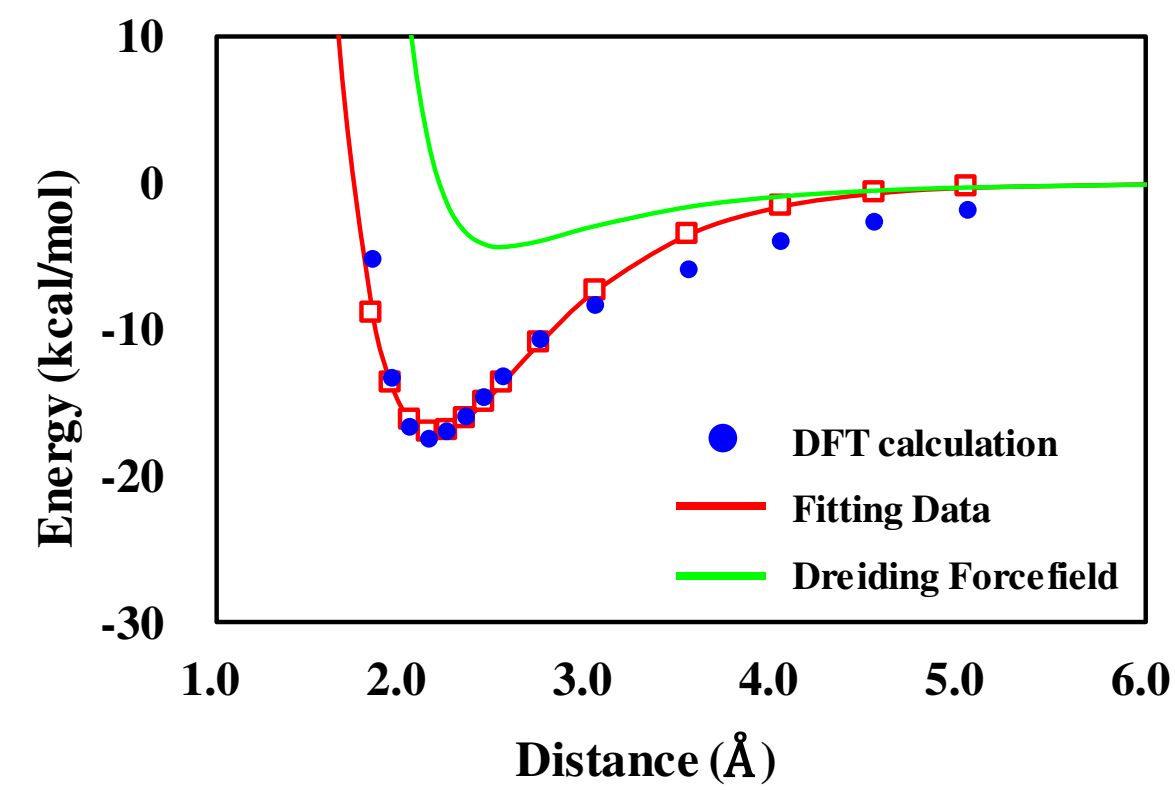

Figure 2. (a) The optimized structures of $\mathrm{O}_{2}$ molecules on the Pt (111) surface. (b) The change in potential energies according to changes in distance between $\mathrm{O}_{2}$ and the $\mathrm{Pt}$ (111) surface, as determined using DFT calculations, fitting data, and DREIDING force field. 
(a) $\lambda=2.92$

Pt (111) Hydrated $\mathrm{O}_{2}$ molecules

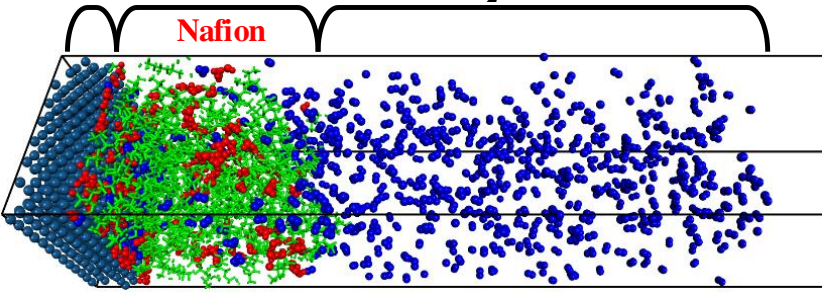

(b) $\lambda=6.15$

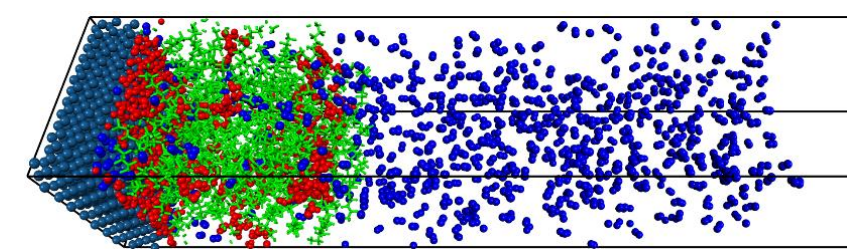

(c) $\lambda=9.77$

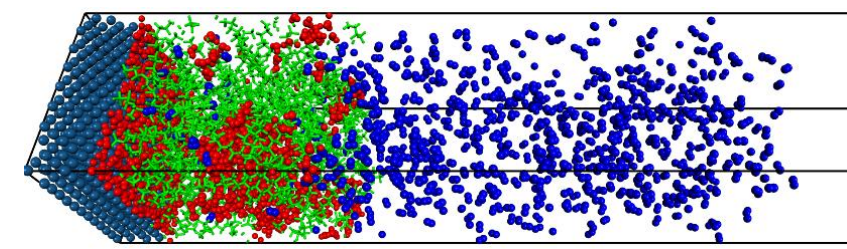

(d)

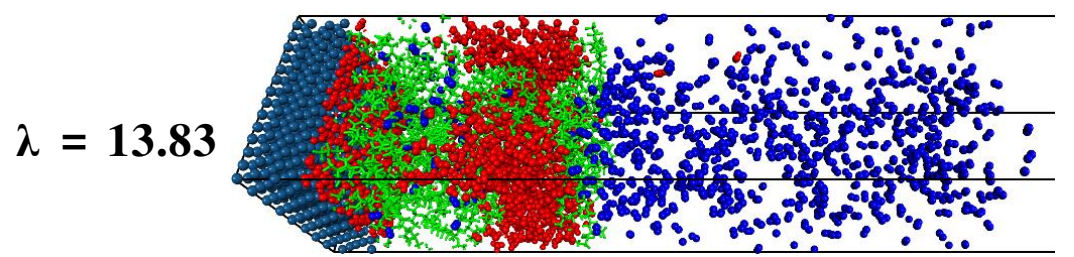

(e)

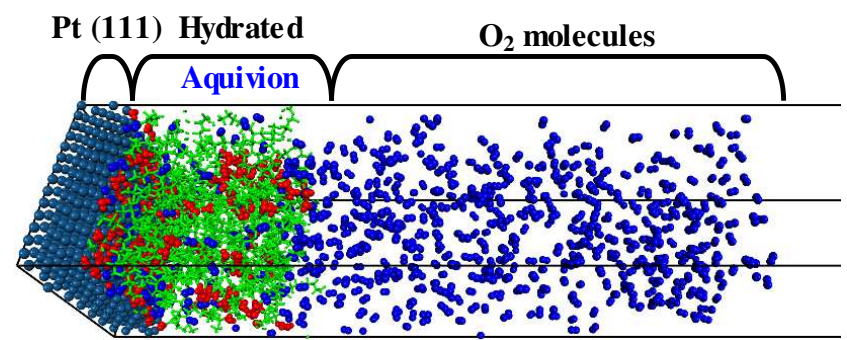

(f)

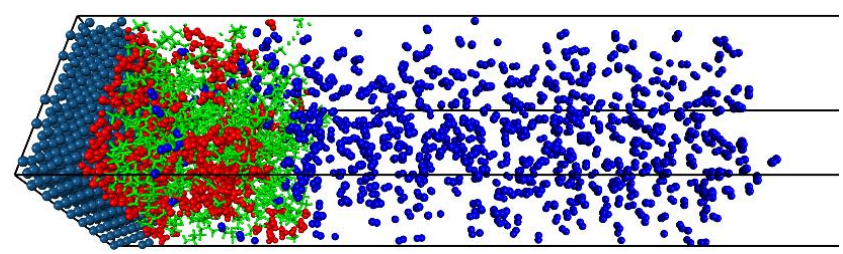

(g)

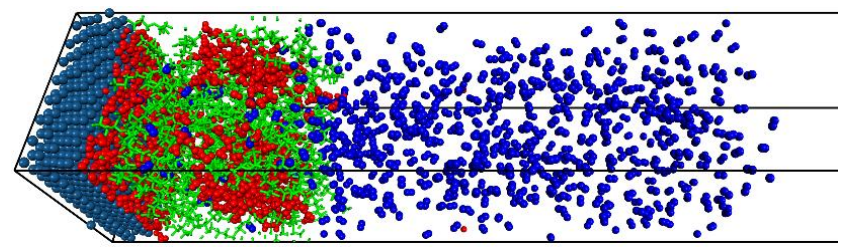

(h)

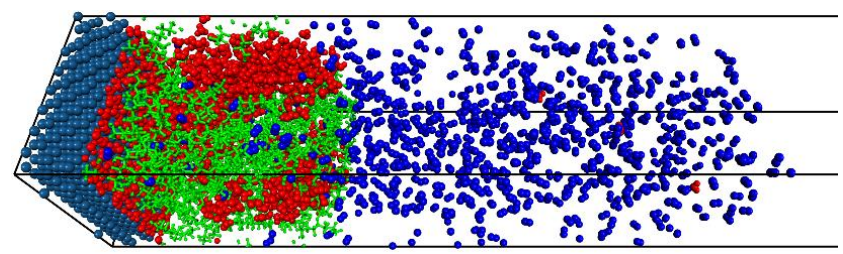

Pt surface

$\mathrm{O}_{2}$ molecules

$\mathrm{H}_{2} \mathrm{O}$ and $\mathrm{H}_{3} \mathrm{O}^{+}$

Figure 3. Snapshots of equilibrated systems comprising the $\mathrm{Pt}$ (111) surface, hydrated Nafion ionomers, and $\mathrm{O}_{2}$ molecules with $\lambda$ values of (a) 2.92 , (b) 6.15 , (c) 9.77, and (d) 13.83; snapshots of equilibrated systems comprising the $\mathrm{Pt}$ (111) surface, hydrated Aquivion ionomers, and $\mathrm{O}_{2}$ molecules with $\lambda$ values of (e) 2.92 , (f) 6.15 , (g) 9.77 , and (h) 13.83 . 
(a)

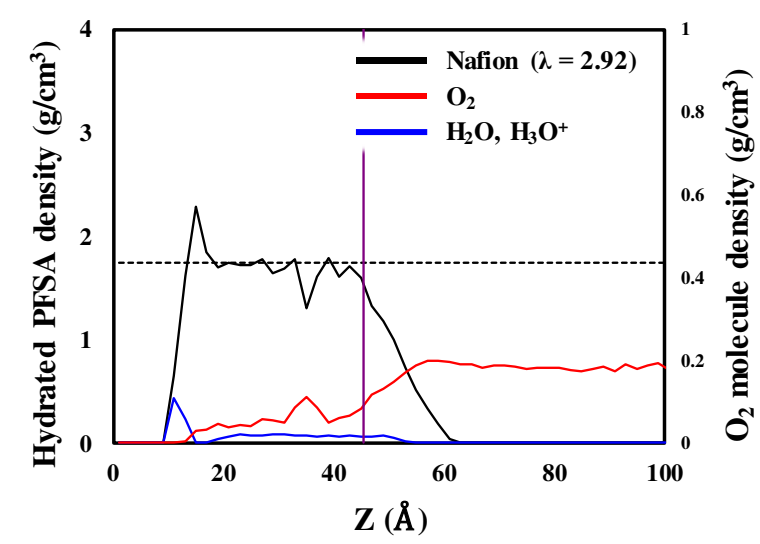

(c)

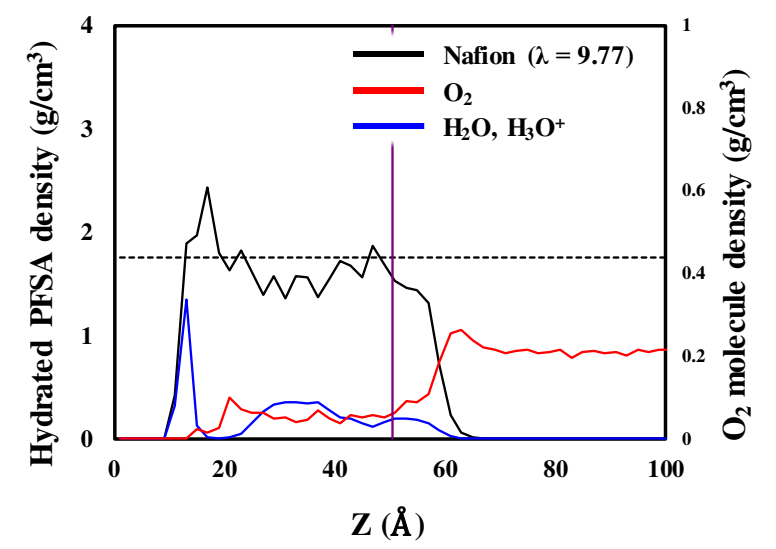

(b)

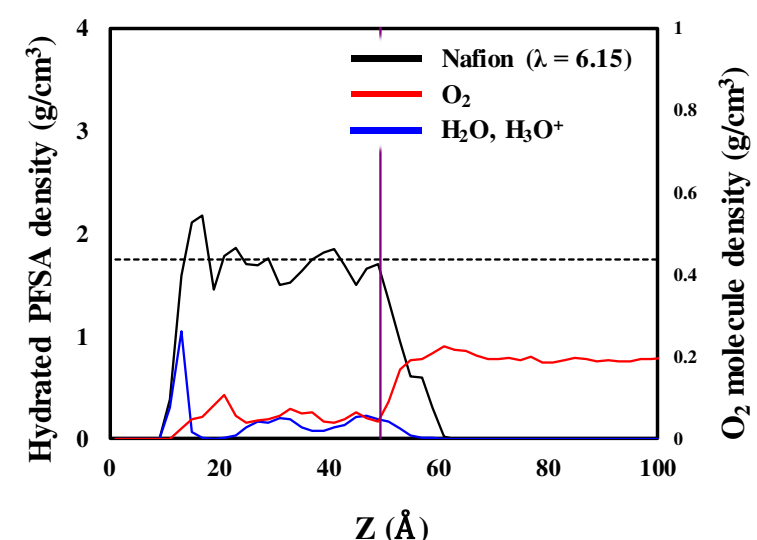

(d)

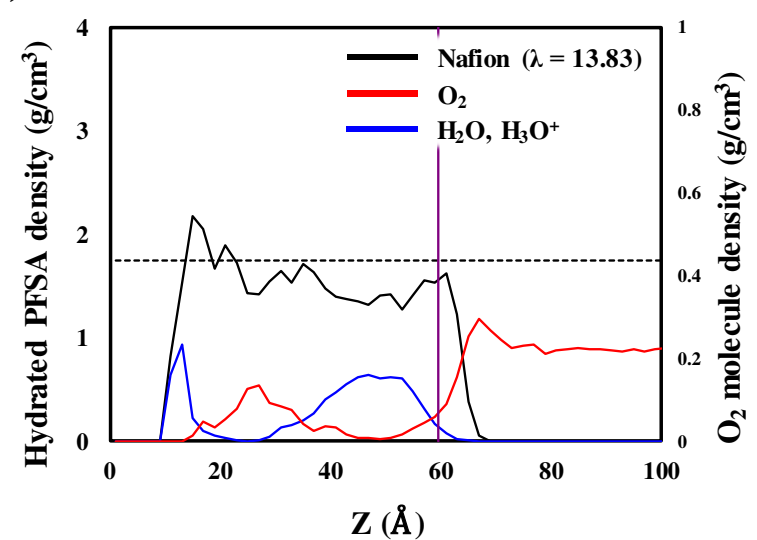

Figure 4. Density distributions of hydrated Nafion ionomer (with included water and hydronium ions; black lines), water and hydronium ions (blue lines), and $\mathrm{O}_{2}$ molecules (red lines) on the Pt (111) surface along the thickness direction, with $\lambda$ values of (a) 2.92, (b) 6.15, (c) 9.77, and (d) 13.83. Dashed lines show the previously reported density for hydrated bulk Nafion. ${ }^{20}$ The purple line indicates the point of $\mathrm{O}_{2}$ solvation occurs from the interface of gas/hydrated Nafion. 
(a)

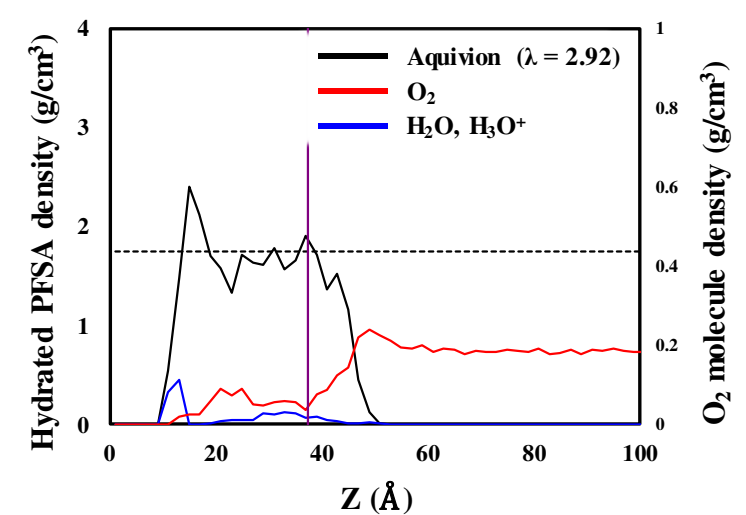

(c)

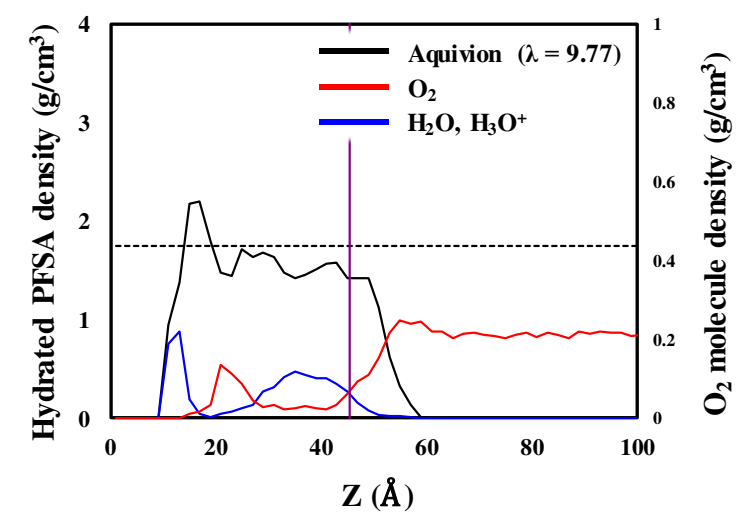

(b)

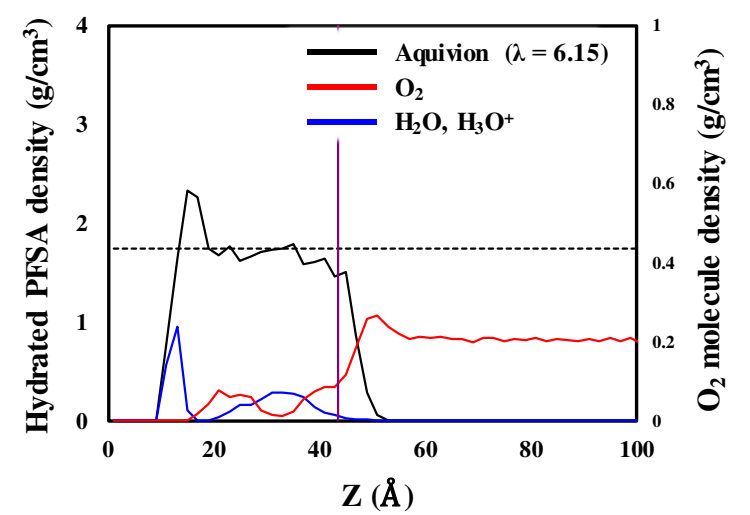

(d)

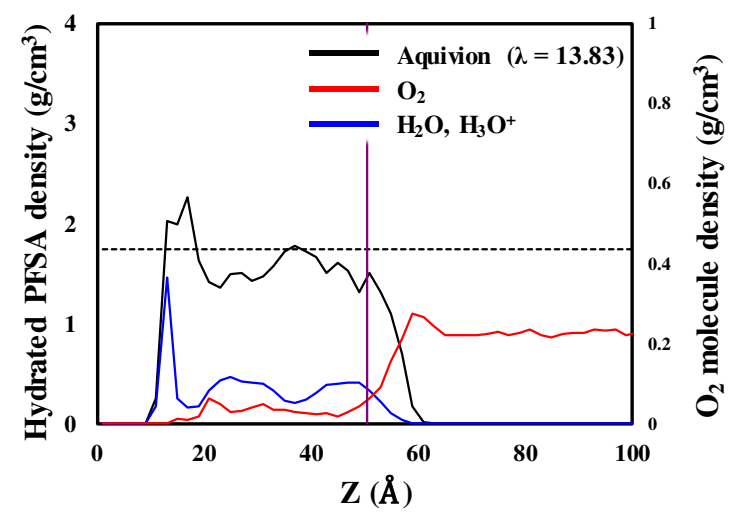

Figure 5. Density distributions of hydrated Aquivion ionomer (with included water and hydronium ions; black lines), water and hydronium ions (blue lines), and $\mathrm{O}_{2}$ molecules (red lines) on the Pt (111) surface along the thickness direction, with $\lambda$ values of (a) 2.92, (b) 6.15, (c) 9.77, and (d) 13.83. Dashed lines show the previously reported density for hydrated bulk Aquivion. ${ }^{20}$ The purple line indicates the point of $\mathrm{O}_{2}$ solvation occurs from the interface of gas/hydrated Nafion. 
(a)

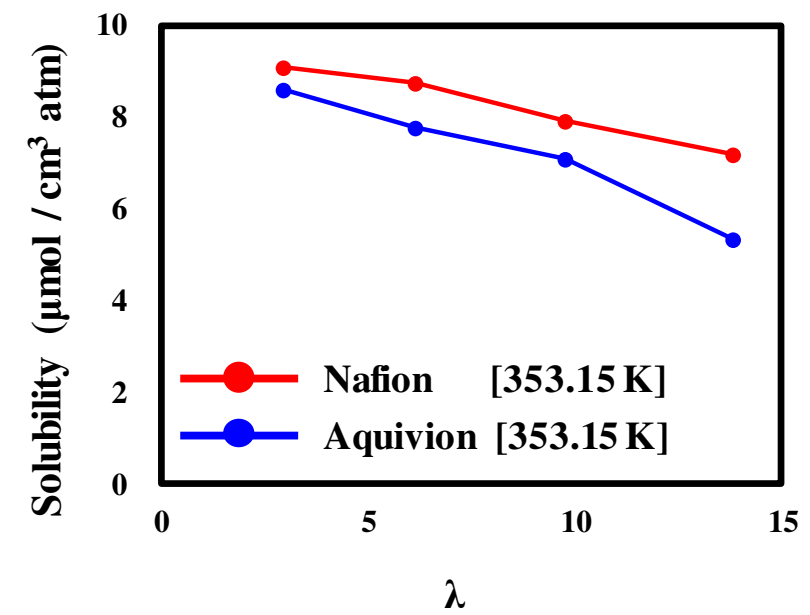

(b)

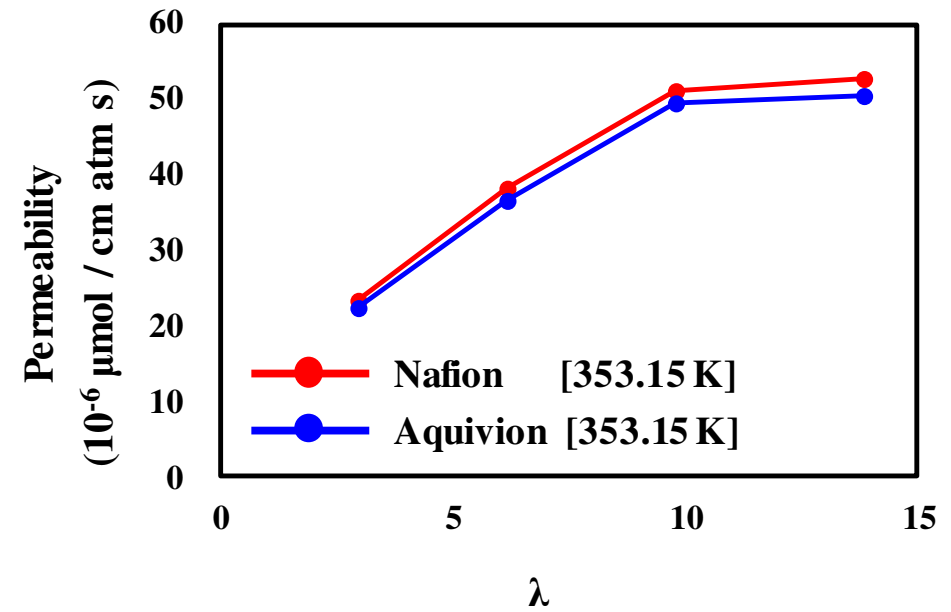

Figure 6. $\mathrm{O}_{2}$ (a) solubility and (b) permeability in hydrated Nafion and Aquivion on a $\mathrm{Pt}(111)$ surface. 
(b)

(a)

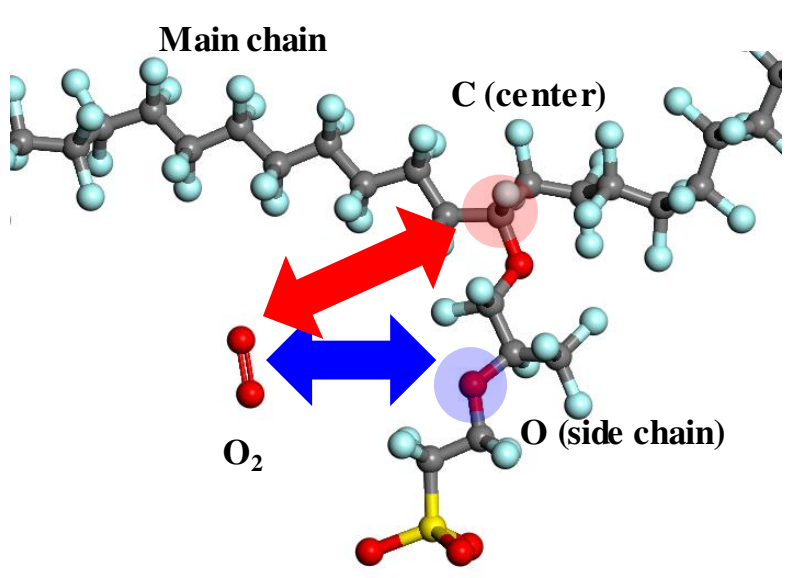

Side chain

(d)

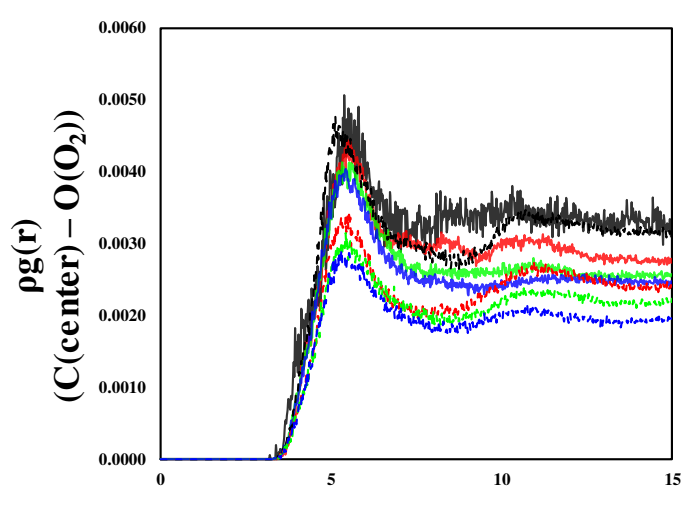

$\lambda$

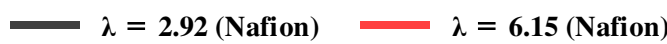

$-\because \lambda=2.92$ (Aquivion) $--=\lambda=6.15$ (Aquivion)

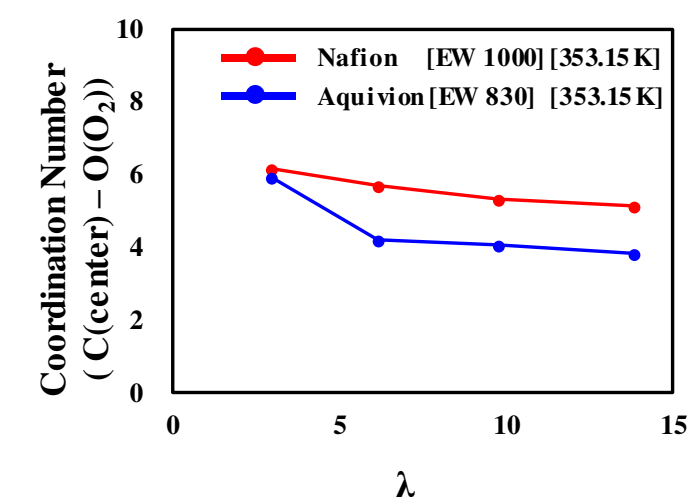

(c)

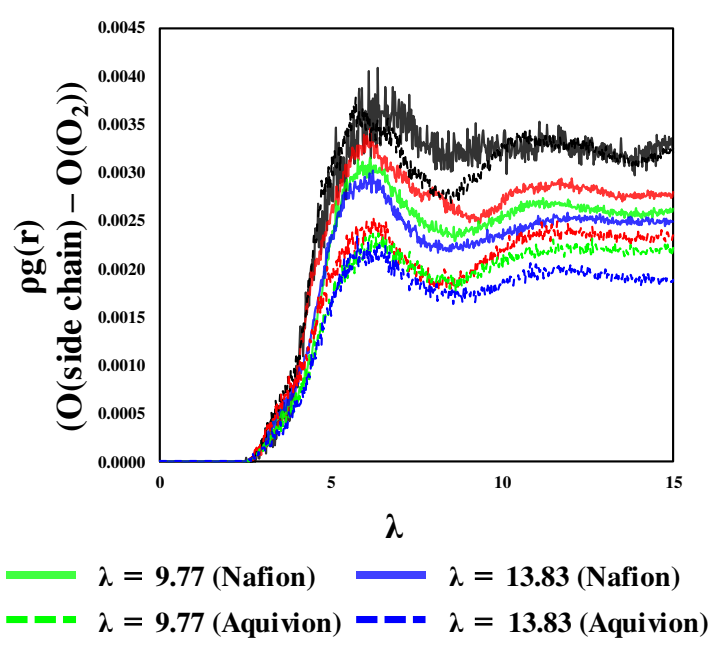

(e)

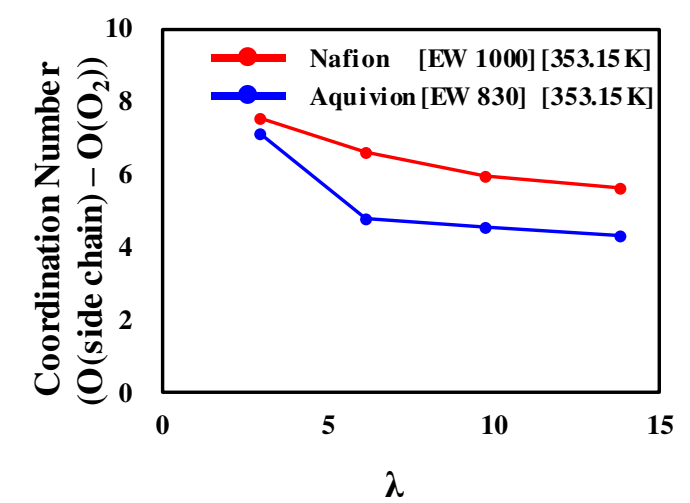

Figure 7. (a) Molecular model with the atomic definition for $\mathrm{O}_{2}$ location analysis using RDFs. $\mathrm{RDFs}$ at (b) $\mathrm{C}\left(\right.$ center) $-\mathrm{O}\left(\mathrm{O}_{2}\right)$ and (c) $\mathrm{O}$ (side chain)- $\mathrm{O}\left(\mathrm{O}_{2}\right)$ and the first coordination number of (d) $\mathrm{C}($ center $)-\mathrm{O}\left(\mathrm{O}_{2}\right)$ and (e) $\mathrm{O}$ (side chain) $-\mathrm{O}\left(\mathrm{O}_{2}\right)$. 
Figures

(a)

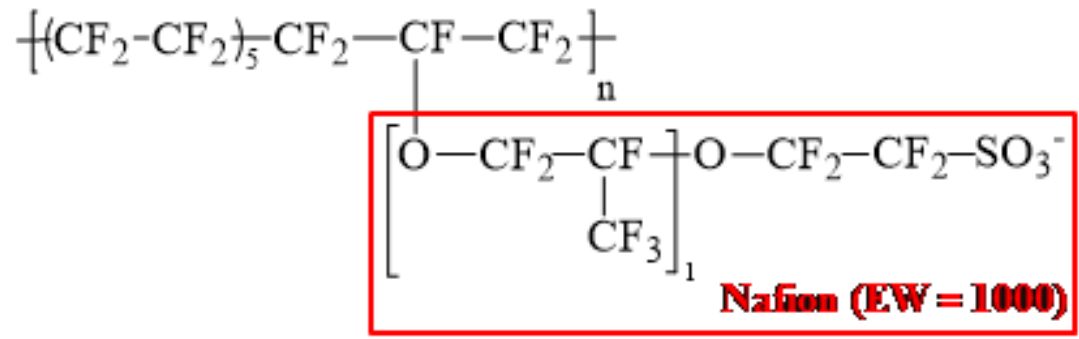

(b) (c)<smiles>O</smiles>

(d)<smiles>O</smiles>

(e)

Figure 1

Chemical structures of PFSA component at (a) ionized Nafion $(E W=1000)$, (b) ionized Aquivion (EW = 830), and (c) water, (d) hydronium ion, and (e) 02 molecules.

(a)

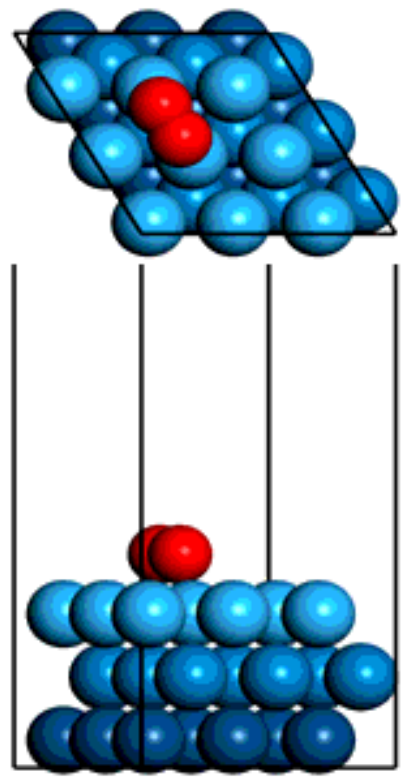

(b)

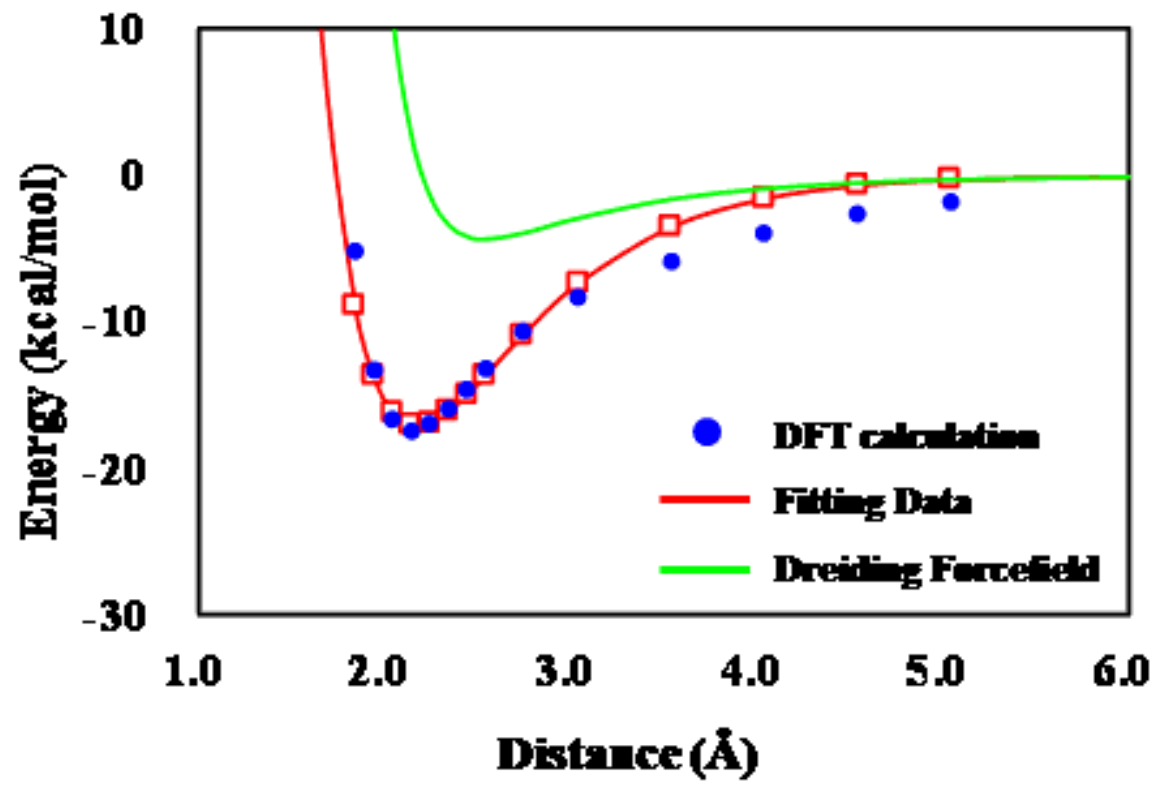

Figure 2

(a) The optimized structures of 02 molecules on the Pt (111) surface. (b) The change in potential energies according to changes in distance between 02 and the Pt (111) surface, as determined using DFT calculations, fitting data, and DREIDING force field. 
(a) $\lambda=2.92$
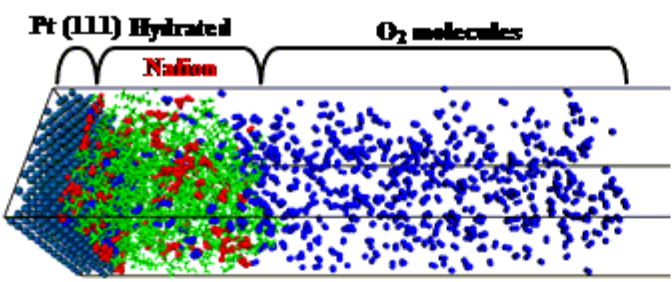

(b) $\lambda=6.15$

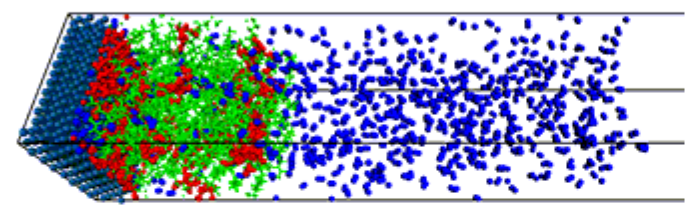

(c) $\lambda=9.77$

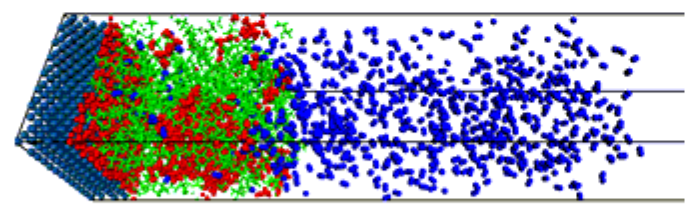

(d)

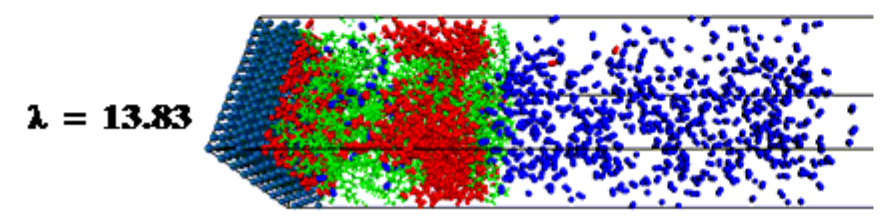

(e)

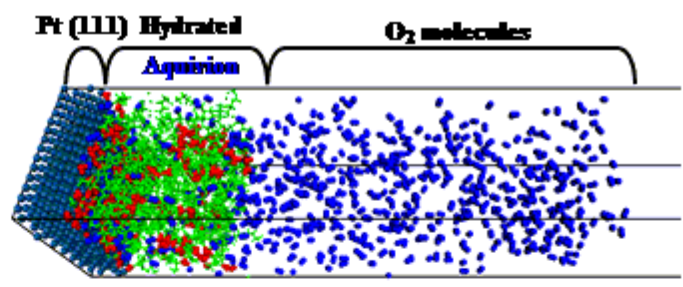

(I)

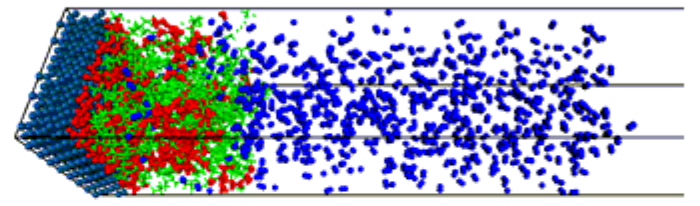

(g)

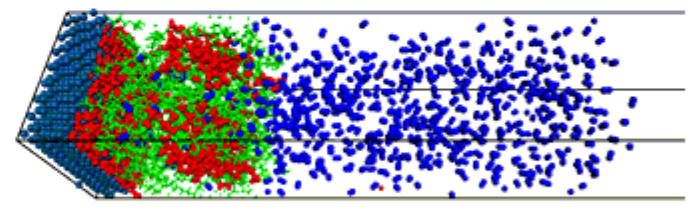

(h)

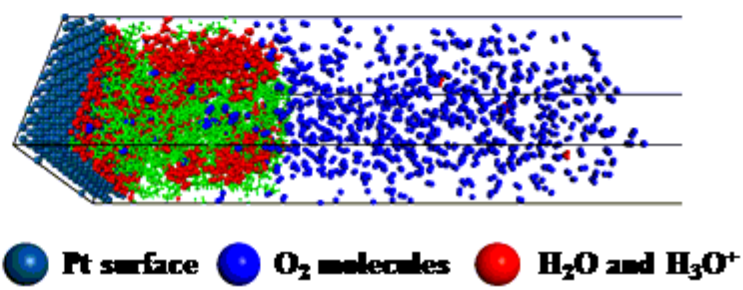

Figure 3

Snapshots of equilibrated systems comprising the Pt (111) surface, hydrated Nafion ionomers, and 02 molecules with $\lambda$ values of (a) 2.92, (b) 6.15 , (c) 9.77 , and (d) 13.83; snapshots of equilibrated systems comprising the $\mathrm{Pt}$ (111) surface, hydrated Aquivion ionomers, and 02 molecules with $\lambda$ values of (e) 2.92 , (f) $6.15,(g) 9.77$, and (h) 13.83 . 
(a)

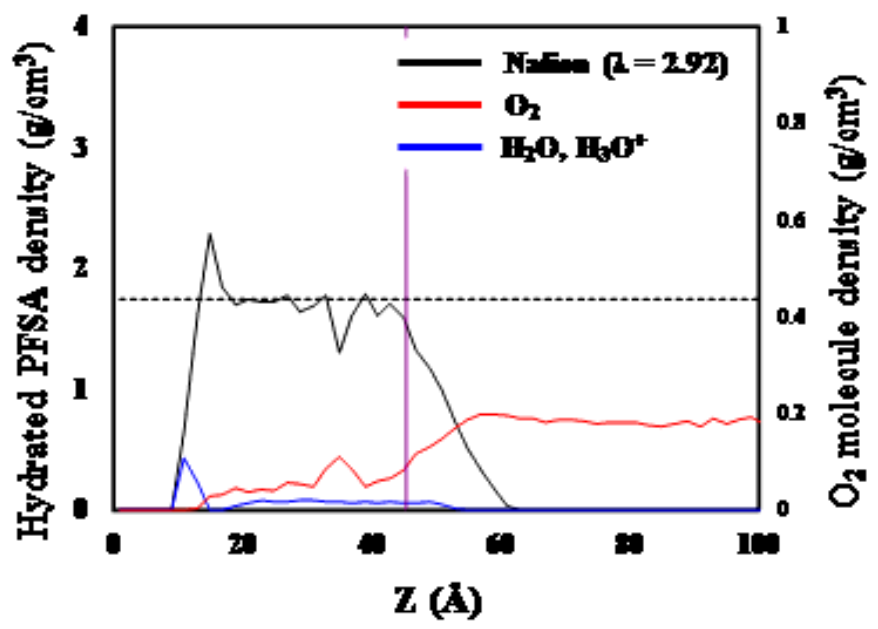

(c)

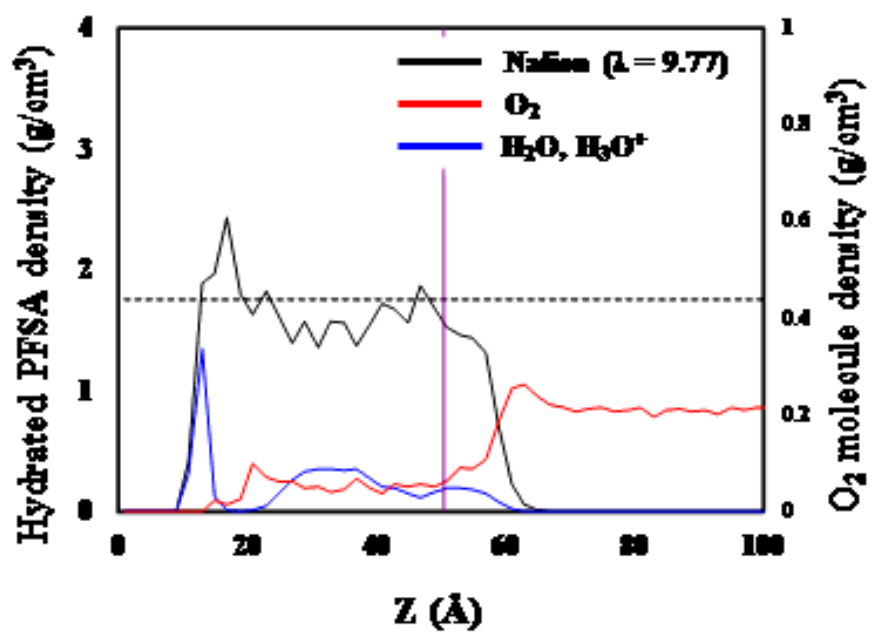

(b)

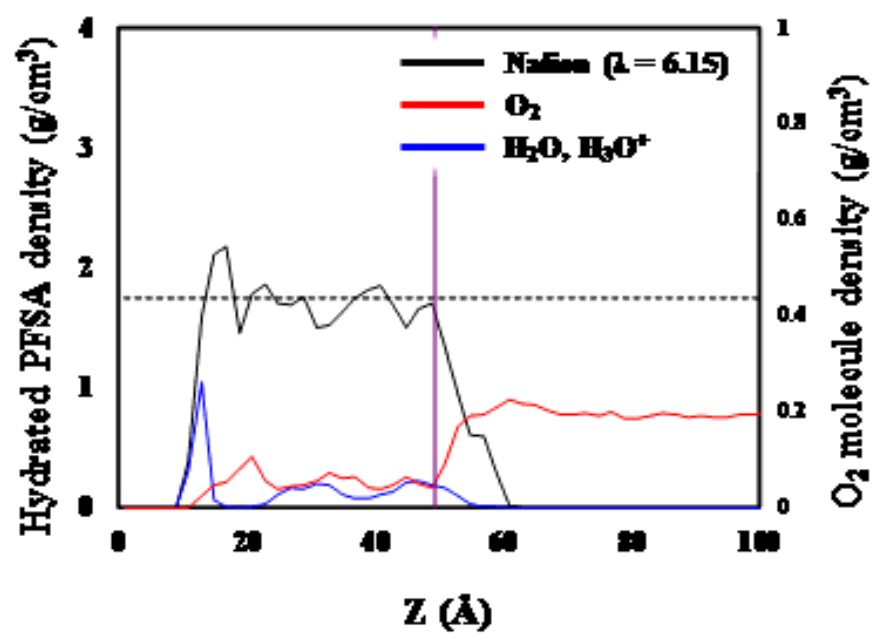

(d)

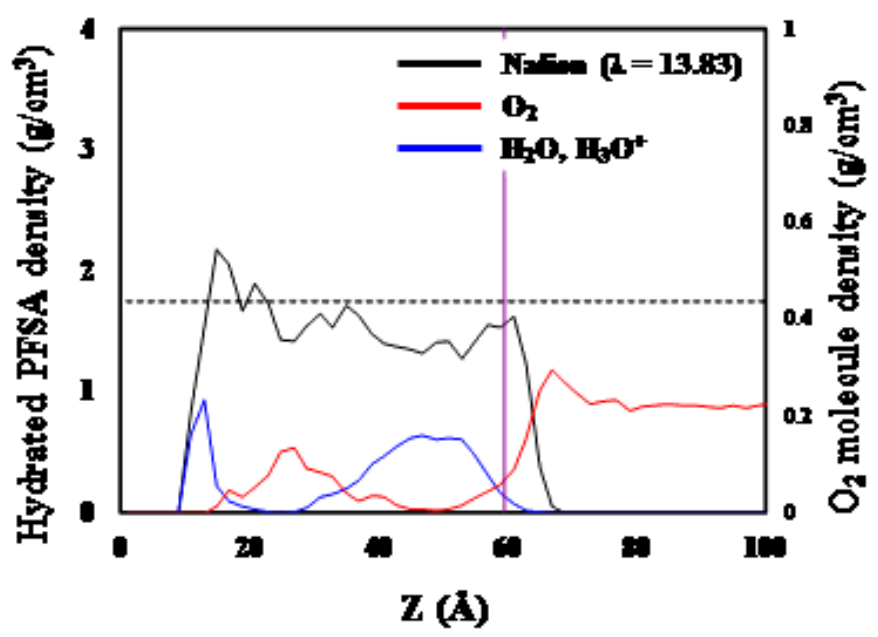

Figure 4

Density distributions of hydrated Nafion ionomer (with included water and hydronium ions; black lines), water and hydronium ions (blue lines), and 02 molecules (red lines) on the Pt (111) surface along the thickness direction, with $\lambda$ values of (a) 2.92, (b) 6.15, (c) 9.77, and (d) 13.83. Dashed lines show the previously reported density for hydrated bulk Nafion.20 The purple line indicates the point of 02 solvation occurs from the interface of gas/hydrated Nafion. 
(a)

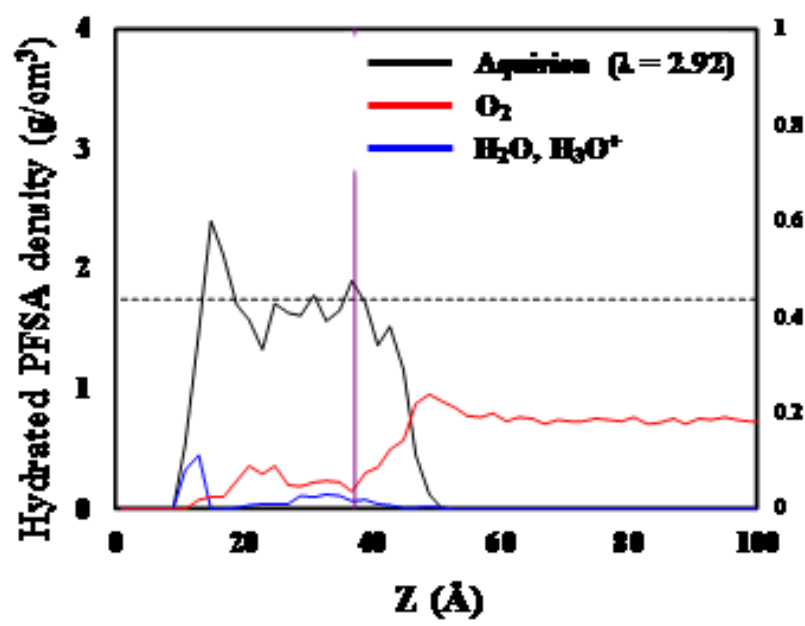

(c)

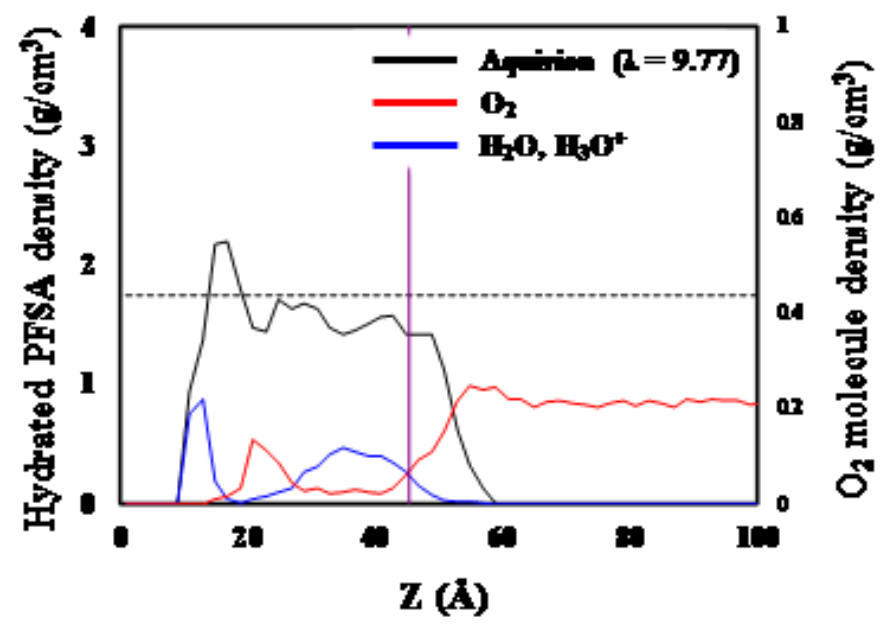

(b)

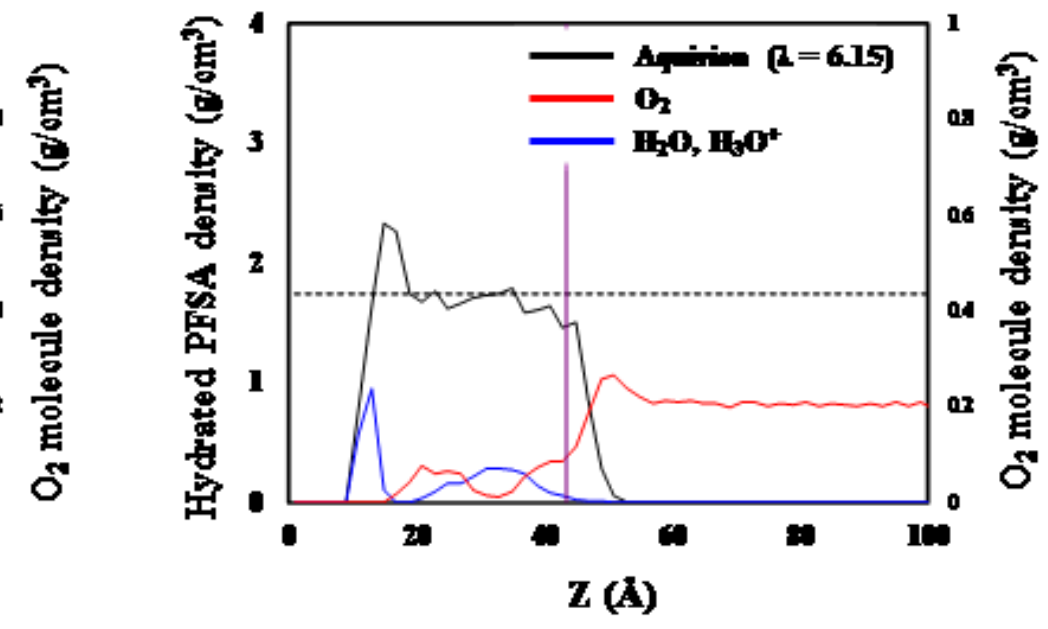

(d)

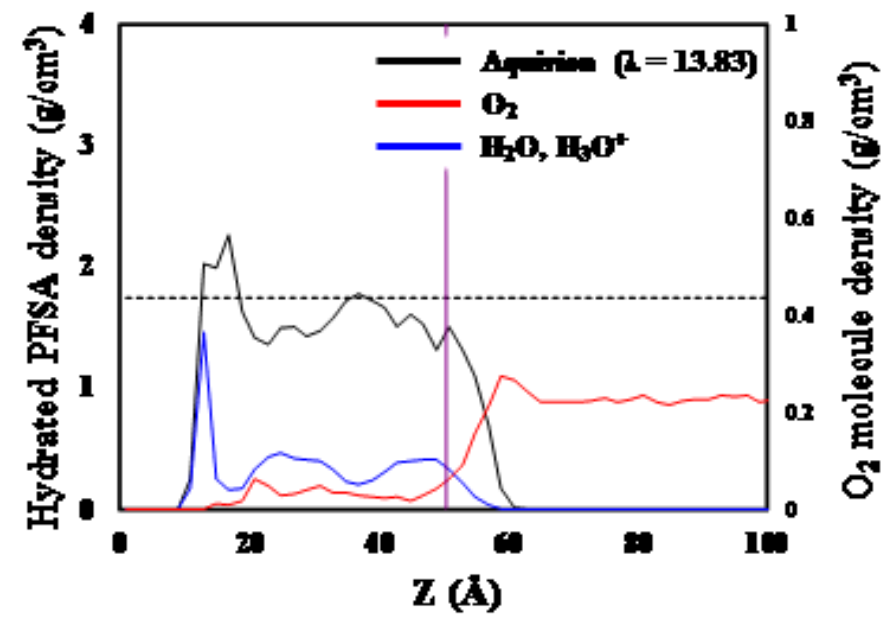

Figure 5

Density distributions of hydrated Aquivion ionomer (with included water and hydronium ions; black lines), water and hydronium ions (blue lines), and 02 molecules (red lines) on the Pt (111) surface along the thickness direction, with $\lambda$ values of (a) 2.92, (b) 6.15, (c) 9.77, and (d) 13.83. Dashed lines show the previously reported density for hydrated bulk Aquivion.20 The purple line indicates the point of 02 solvation occurs from the interface of gas/hydrated Nafion. 
(a)

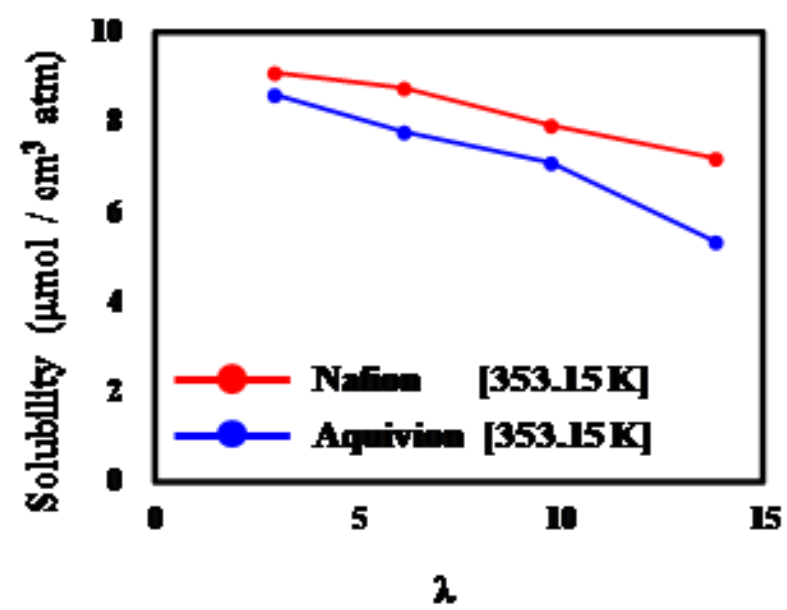

(b)

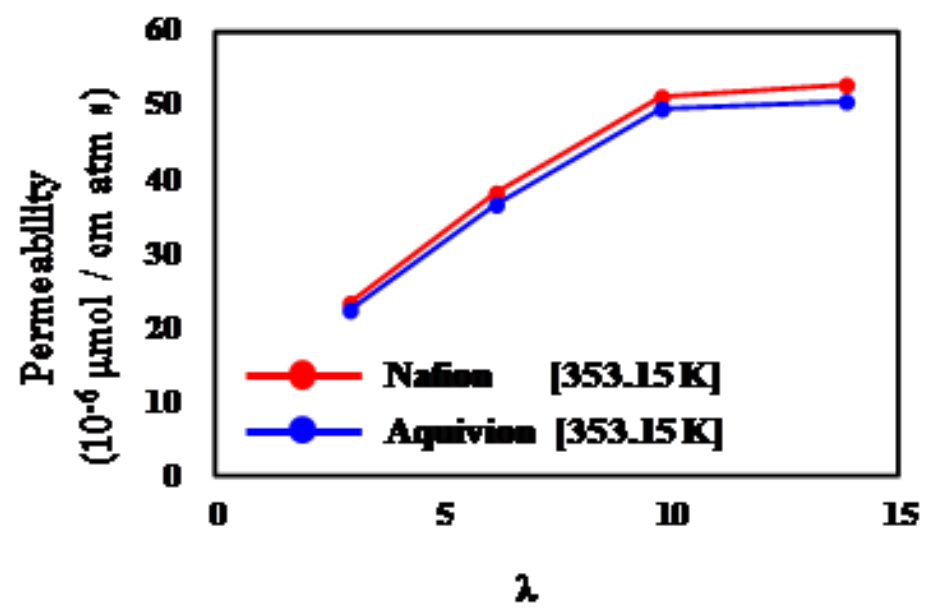

Figure 6

O2 (a) solubility and (b) permeability in hydrated Nafion and Aquivion on a Pt (111) surface.

(b)

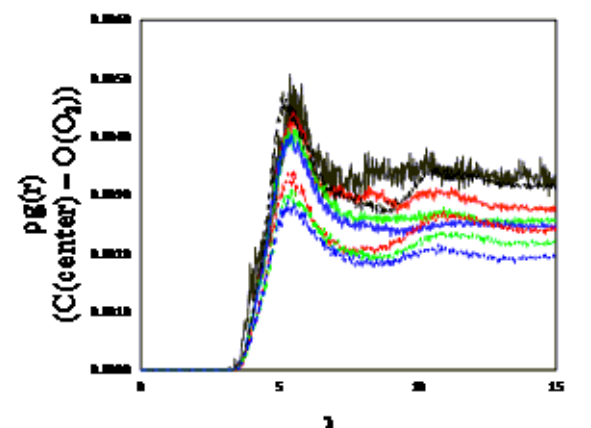

$\boldsymbol{\lambda}$

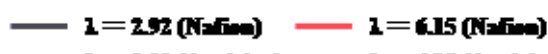

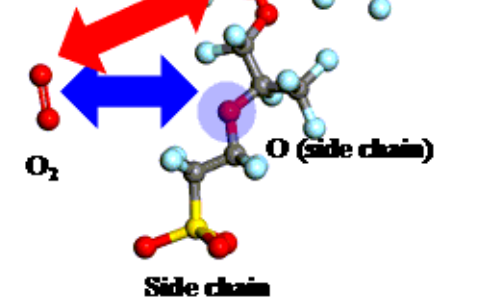

(d) (c)

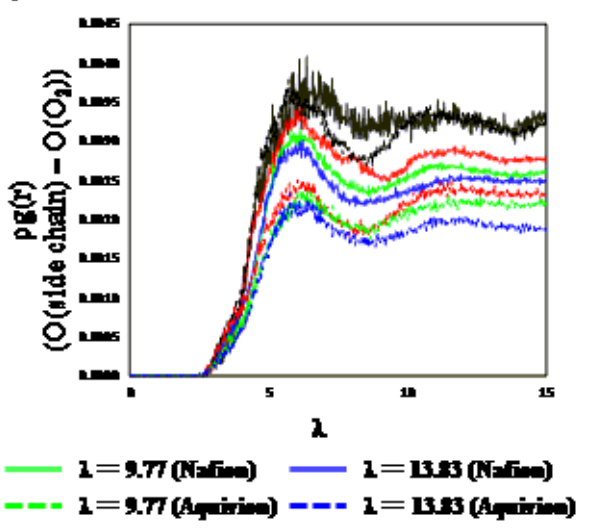

(e)

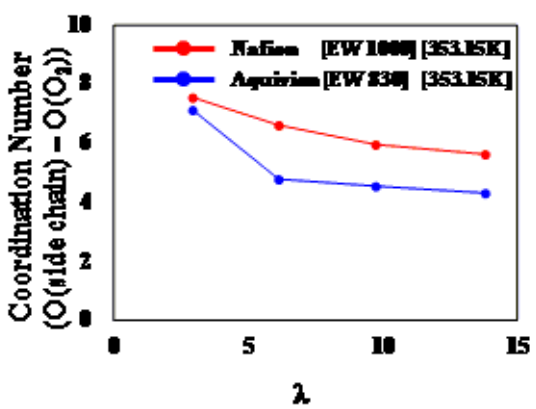

Figure 7

(a) Molecular model with the atomic definition for $\mathrm{O} 2$ location analysis using RDFs. RDFs at (b) $\mathrm{C}$ (center) $-\mathrm{O}(\mathrm{O} 2)$ and (c) $\mathrm{O}$ (side chain) $-\mathrm{O}(\mathrm{O} 2)$ and the first coordination number of (d) $\mathrm{C}$ (center) $-\mathrm{O}(\mathrm{O} 2)$ and (e) $\mathrm{O}$ (side chain) $-\mathrm{O}(02)$. 